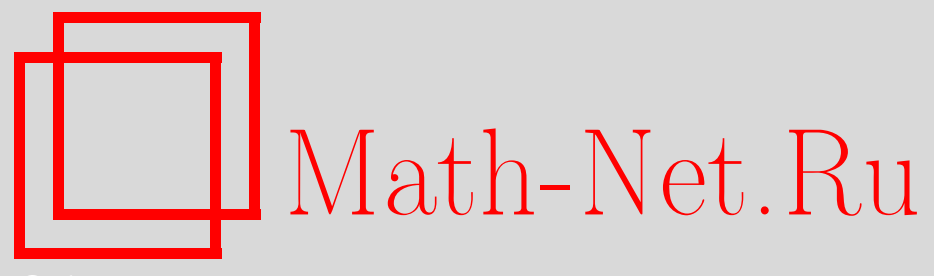

В. П. Маслов, В. Е. Назайкинский, О распределении целочисленных случайных величин, связанных одним линейным неравенством. I, Матем. заметки, 2008, том 83, выпуск 2, 232-263

DOI: https://doi.org/10.4213/mzm4418

Использование Общероссийского математического портала Math-Net.Ru подразумевает, что вы прочитали и согласны с пользовательским соглашением http://www.mathnet.ru/rus/agreement

Параметры загрузки:

IP : 54.224 .60 .19

26 апреля 2023 г., $17: 47: 51$

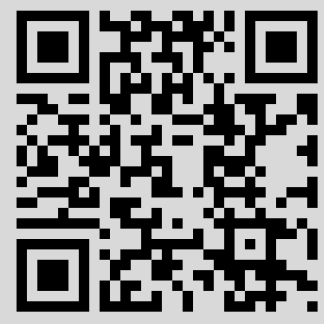


Том 83 выпуск 2 февраль 2008

УДК $519.2+531.19$

\title{
О распределении целочисленных случайных величин, связанных одним линейным неравенством. I
}

\author{
В. П. Маслов, В. Е. Назайкинский
}

Рассматривается математическая задача о распределении тождественных частиц по целочисленным уровням энергии при условии, что число частиц не задано, а суммарная энергия системы ограничена сверху. Рассматриваются системы как целой, так и дробной размерности. Числа заполнения могут принимать либо произвольные целые неотрицательные значения (“бозе-частицы”), либо значения из конечного множества $\{0,1, \ldots, R\}$ (так называемые парастатистики; например, при $R=1$ получается статистика Ферми-Дирака). При условии, что все варианты, удовлетворяющие заданным ограничениям, равновероятны, изучается явление концентрации большинства вариантов при больших энергиях вблизи отвечающего заданной парастатистике предельного распределения.

Библиография: 10 названий.

\section{1. Введение}

В работах [1]-[3] изучена с математической точки зрения задача о кумулятивных вероятностях распределения системы тождественных частиц по уровням энергии при условии, что задано число частиц и ограничение на суммарную энергию системы, а все варианты распределения, удовлетворяющие заданным ограничениям, равновероятны. В данной заметке мы обратимся к случаю, когда число частиц не задано, а ограничение накладывается только на суммарную энергию. Предполагается, что уровни энергии - целые числа. Рассматриваются системы как целой, так и дробной размерности (точные определения даны в основном тексте работы). Числа частиц на уровнях (числа заполнения) могут принимать либо произвольные целые неотрицательные значения (“бозе-частицы”), либо значения из конечного множества $\{0,1, \ldots, R\}$ (так называемые парастатистики, ср. [4]; например, при $R=1$ наши “частицы" подчиняются статистике Ферми-Дирака). Допускается также, чтобы число $R$ зависело от номера уровня энергии. Вычисляются асимптотические кумулятивные распределения и энтропия рассматриваемых систем, получены поправки к интегральным формулам для энергии и числа частиц, аналогичные указанной в [5] поправке порядка $V^{2 / 3}$ для закона Стефана-Больцмана (см. [6]).

1.1. Пример: трехмерная система. Прежде чем описывать общую постановку задачи, решаемой в данной работе, рассмотрим простейший пример. Для этого примера мы, в частности, сформулируем некоторые из основные утверждений, доказанных нами ниже.

(C) В.П. МАслов, В.Е. НАЗАЙКинский, 2008 
Пусть каждой точке $(x, y, z)$ положительного октанта трехмерной целочисленной решетки $\mathbb{Z}^{3}$ (кроме точки $(0,0,0)$ ) сопоставлено каким-то образом произвольное неотрицательное целое число $N_{x y z}, x, y, z \in \mathbb{Z}_{+}=\{0,1, \ldots\}, x+y+z \geqslant 1$. Такой набор чисел обозначим через $\left\{N_{x y z}\right\}$. Рассмотрим всевозможные наборы $\left\{N_{x y z}\right\}$, удовлетворяющие условию

$$
\sum_{x, y, z}(x+y+z) N_{x y z} \leqslant M
$$

где $M>0$ - заданное целое число, а сумма берется по всем указанным выше точкам $(x, y, z)$. Число $\mathscr{N}(M)$ таких наборов конечно, ибо из (1) вытекает, что каждое из чисел $N_{x y z}$ не превосходит $M$, а те из них, для которых $x+y+z>M$, с необходимостью равны нулю.

Все наборы $\left\{N_{x y z}\right\}$, удовлетворяющие условию (1) будем считать равноправными (или, если угодно, равновероятными - можно представить себе, что набор выбирается случайным образом, причем вероятность выбора любого конкретного набора одинакова и равна $1 / \mathscr{N}(M))$.

Хотя сами наборы $\left\{N_{x y z}\right\}$ случайны и равновероятны (а точнее, именно благодаря этому обстоятельству), при больших $M$ некоторые "агрегированные" характеристики этих наборов с большой вероятностью располагаются вблизи некоторых вполне определенных значений. Одной из таких характеристик является сумма $\sum_{x, y, z} N_{x y z}$ всех чисел $N_{x y z}$. Оказывается, эта сумма для большинства вариантов близка к числу $N$, которое определяется следующим образом:

$$
N=\sum_{j=1}^{\infty} \frac{(j+1)(j+2)}{2\left(e^{b j}-1\right)},
$$

где $b$ - решение уравнения

$$
M=\sum_{j=1}^{\infty} \frac{j(j+1)(j+2)}{2\left(e^{b j}-1\right)} .
$$

(Это решение всегда существует, единственно и стремится к нулю при $M \rightarrow \infty$, так что $N \rightarrow \infty$ при $M \rightarrow \infty$. Более того, $N$ и $M$ имеют порядок $b^{-3}$ и $b^{-4}$ соответственно.) Именно, зафиксируем произвольную положительную функцию $\psi(x)$, которая стремится к $+\infty$ при $x \rightarrow+\infty$ (например, итерированный сколько угодно раз логарифм $\psi(x)=\ln [\ln [\ldots \ln [x] \ldots]])$ и положим

$$
\Delta N=\sqrt{N \ln N} \psi(N)
$$

(Таким образом, с точностью до произвольно медленно растущего множителя величина $\Delta N$ есть просто $\sqrt{N \ln N}$.) Тогда справедливо следующее утверждение.

Теорема 1. Существуют такие постоянные $C_{k}, k=1,2, \ldots$, что

$$
\mathrm{P}\left(\left|\sum_{x, y} N_{x y z}-N\right|>\Delta N\right) \leqslant C_{k} N^{-k} .
$$


Разумеется, вероятность в левой части формулы (5) определяется как отношение числа $\mathscr{N}(M, \Delta N)$ наборов $\left\{N_{x y z}\right\}$, удовлетворяющих условию (1) и таких, что

$$
\left|N-\sum N_{x y z}\right|>\Delta N
$$

к общему числу $\mathscr{N}(M)$ наборов $\left\{N_{x y z}\right\}$, удовлетворяющих условию $(1)$ :

$$
\mathrm{P}\left(\left|\sum_{x, y, z} N_{x y z}-N\right|>\Delta N\right):=\frac{\mathscr{N}(M, \Delta N)}{\mathscr{N}(M)} .
$$

Другая характеристика - это сумма $\sum_{x, y, z}(x+y+z) N_{x y z}$. Из условия задачи известно лишь, что она не превосходит $M$ (и, разумеется, неотрицательна, поскольку все $N_{x y z}$ неотрицательны). Оказывается, однако, что на самом деле для подавляющего большинства вариантов эта сумма весьма близка к $M$. Именно, положим

$$
\Delta M=\frac{M}{N} \Delta N
$$

Теорема 2. Существуют такие постоянные $C_{k}, k=1,2, \ldots$, что

$$
\mathrm{P}\left(\left|\sum_{x, y, z}(x+y+z) N_{x y z}-M\right|>\Delta M\right) \leqslant C_{k} N^{-k} .
$$

Утверждения теорем 1 и 2 остаются верными и в том случае, если суммирование по $(x, y, z)$ распространяется не на все точки, а лишь на некоторое подмножество целочисленной решетки (причем постоянные $C_{k}$ не будут зависеть от конкретного выбора подмножества). При этом, конечно, $N$ и $M$ также следует заменить на соответствующие части сумм, входящих в формулы (2) и (3). Произвольные подмножества нас, впрочем, мало интересуют, и мы сформулируем соответствующие утверждения в нужном нам случае. Совокупность целочисленных точек $(x, y, z)$ из положительного октанта, для которых

$$
x+y+z=j, \quad j=1,2, \ldots,
$$

назовем $j$-м уровнем энергии, а число $q_{j}$ таких точек на этом уровне - его кратностью (нетрудно видеть, что $\left.q_{j}=(j+1)(j+2) / 2\right)$. Обозначим теперь через $N_{j}$ сумму всех чисел $N_{x y z}$, находящихся на $j$-м уровне энергии:

$$
N_{j}=\sum_{x+y+z=j} N_{x y z} .
$$

Теорема 3. Существуют такие постояннъе $C_{k}, k=1,2, \ldots$, что для любого $l \in \mathbb{Z}_{+}$справедливь оценки

$$
\begin{gathered}
\mathrm{P}\left(\left|\sum_{j=1}^{l} N_{j}-\sum_{j=1}^{l} \frac{(j+1)(j+2)}{2\left(e^{b j}-1\right)}\right|>\Delta N\right) \leqslant C_{k} N^{-k}, \\
\mathrm{P}\left(\left|\sum_{j=1}^{l} j N_{j}-\sum_{j=1}^{l} \frac{j(j+1)(j+2)}{2\left(e^{b j}-1\right)}\right|>\Delta M\right) \leqslant C_{k} N^{-k} .
\end{gathered}
$$


Оценим теперь энтропию $S$ рассматриваемой системы. Под энтропией в данной работе будем понимать логарифм числа $\mathscr{N}(M)$ всевозможных целочисленных неотрицательных наборов $\left\{N_{x y z}\right\}$, удовлетворяющих ограничению (1):

$$
S=\ln \mathscr{N}(M) .
$$

(Определение, связанное с колмогоровской сложностью, используется в [7]; здесь мы эти вопросы не затрагиваем.)

Теорема 4. При $M \rightarrow \infty$ справедлива асимптотическая формула

$$
S \equiv \ln \mathscr{N}(M)=b M-\sum_{j=1}^{\infty} \frac{(j+1)(j+2)}{2} \ln \left(1-e^{-b j}\right)+O(\ln N) .
$$

Закончим рассмотрение примера, вернувшись к формулам (2) и (3). Эти формулы задают $M$ и $N$ как суммы некоторых рядов. Оказывается, однако, что эти ряды можно приблизить интегралами, интегральными суммами которых они являются. Справедливо следующее предложение.

ПреДЛОЖЕНИЕ 5. Пусть $M$ и $N$ заданы формулами (2) и (3). Тогда при $M \rightarrow \infty$ справедливы соотношения

$$
\begin{aligned}
& N=\int_{0}^{\infty} \frac{x^{2} d x}{2\left(e^{b x}-1\right)}+O\left(N^{2 / 3}\right), \\
& M=\int_{0}^{\infty} \frac{x^{3} d x}{2\left(e^{b x}-1\right)}+O(N) .
\end{aligned}
$$

Точность выражений (12) и (13), однако, недостаточна для того, чтобы в теоремах 1-4 можно было заменить суммы (2) и (3) на интегралы (12) и (13). К ним нужно добавить последующие члены разложения, полученные из формулы Эйлера-Маклорена. Соответствующие результаты описаны ниже в п. 1.3.3.

1.2. Постановка задачи. Отталкиваясь от описанного выше примера, рассмотрим теперь общую постановку задачи для системы произвольной размерности и при возможном наличии ограничений типа "парастатистики".

1.2.1. Системы целой положительной размерности. Рассмотрим положительный квадрант $d$-мерной целочисленной решетки $\mathbb{Z}^{d}$ и каждой его точке $\left(j_{1}, \ldots, j_{d}\right)$ (кроме точки 0) сопоставим неотрицательное целое число

$$
N_{j_{1} \ldots j_{d}} \in\left\{0,1, \ldots, R_{j_{1}+\cdots+j_{d}}\right\} .
$$

Здесь каждое из чисел $R_{j}>0, j=1,2, \ldots-$ либо целое положительное, либо $+\infty$ (в последнем случае соответствующее число $N_{j_{1} \ldots j_{d}}$ может быть любым целым неотрицательным).

Рассмотрим всевозможные такие наборы

$$
\left\{N_{j_{1} \ldots j_{d}}\right\}, \quad j_{1}, \ldots, j_{d} \in \mathbb{Z}_{+}=\{0,1,2, \ldots\}, \quad j_{1}+\cdots+j_{d}>0,
$$

удовлетворяющие условию

$$
\sum_{j_{1}, \ldots, j_{d}}\left(j_{1}+\cdots+j_{d}\right) N_{j_{1} \ldots j_{d}} \leqslant M
$$


где $M>0$ - заданное целое число, а сумма берется по всем значениям индексов $j_{1}, \ldots, j_{d}$, указанных в (14). По тем же причинам, что и в рассмотренном выше примере, число $\mathscr{N}(M)$ таких наборов конечно. Считая их равноправными (равновероятными), поставим следующие задачи:

1) вычислить значение $N$, около которого концентрируется большинство значений суммы $\sum_{j_{1}, \ldots, j_{d}} N_{j_{1} \ldots j_{d}}$, и доказать соответствующую оценку, аналогичную полученной в теореме 1 , а также получить подобную оценку для $M$;

2) найти асимптотические кумулятивные распределения, аналогичные указанным в теореме 3 , по уровням энергии;

3) найти асимптотическую формулу для энтропии $S=\ln \mathscr{N}(M)$.

1.2.2. Системы произвольной размерности. Чтобы обобщить указанную выше постановку на случай дробных "размерностей" $d$, переформулируем ее так, чтобы избавиться от непосредственного упоминания решетки $\mathbb{Z}^{d}$ и индексов $j_{1}, \ldots, j_{d}$. Именно, пусть $j$ - некоторый уровень энергии, и пусть $q_{i}$ - его кратность, т.е. число различных наборов неотрицательных целых чисел $j_{1}, \ldots, j_{d}$, для которых справедливо соотношение

$$
j=j_{1}+\cdots+j_{d}
$$

Перенумеруем все числа $N_{j_{1} \ldots j_{d}}$, лежащие на одном и том же уровне энергии $j$, в произвольном фиксированном порядке от 1 до $q_{j}$ и будем в дальнейшем обозначать их через $N_{j k}, k=1, \ldots, q_{i}$. Далее, через $N_{j}$ обозначим сумму всех $N_{j k}$ для заданного уровня энергии $j$,

$$
N_{j}=\sum_{k=1}^{q_{j}} N_{j k} .
$$

Теперь $N_{j k} \in\left\{0,1, \ldots, R_{j}\right\}$, а условие (15) можно переписать в виде

$$
\sum_{j=1}^{\infty} j N_{j} \equiv \sum_{j=1}^{\infty} j \sum_{k=1}^{q_{i}} N_{j k} \leqslant M,
$$

и чтобы обобщить постановку задачи на произвольные $d$, осталось выяснить, каким образом можно задавать в таком случае кратности $q_{i}$. Напомним прежде всего формулу для $q_{j}$ при натуральных $d$ :

$$
q_{j}=\frac{(j+d-1) !}{(d-1) ! j !} \equiv C_{j+d-1}^{j} .
$$

Правую часть выражения (18) можно записать через Г-функцию в виде

$$
q_{j}=\frac{\Gamma(j+d)}{\Gamma(d) \Gamma(j+1)} .
$$

Это выражение представляет собой аналитическую функцию от $d$, т.е. задает "аналитическое продолжение" выражения (18) для $q_{i}$ по параметру $d$. Этим мы и воспользуемся, определяя кратности для значений $d$, не являющихся натуральными числами.

В данной работе мы всюду считаем, что $d \geqslant 1$. Случай $d<1$ (и, в частности, случай отрицательной размерности) будет рассмотрен в другом месте (см. по этому поводу также [8]). 
При дробном $d$ выражение (19) непосредственно использовать нельзя, поскольку кратности должны быть целыми числами. Поэтому положим

$$
q_{j}=\left[\frac{\Gamma(j+d)}{\Gamma(d) \Gamma(j+1)}\right], \quad l=1,2, \ldots,
$$

где квадратные скобки обозначают целую часть числа.

1.3. Основные результаты. Перейдем к изложению результатов. Доказательства даны в последующих параграфах.

1.3.1. Определение системы и энтропия. Будем рассматривать систему размерности $d \geqslant 1$, определяемую следующим образом.

Для уровней энергии $j=1,2, \ldots$ кратностей (20) и заданных максимальных "заселенностей" $R_{j} \in\{1,2, \ldots\} \cup\{\infty\}$ каждого из подуровней рассматриваем всевозможные наборы неотрицательных чисел $N_{j k}, k=1, \ldots, q_{j}$, таких, что

$$
\begin{gathered}
\left.N_{j k} \in\left\{0,1, \ldots, R_{j}\right\} \quad \text { (или } N_{j k} \in\{0,1,2, \ldots\}, \text { если } R_{j}=\infty\right), \\
\sum_{j=1}^{\infty} j \sum_{k=1}^{q_{j}} N_{j k} \leqslant M .
\end{gathered}
$$

Все такие наборы считаем равновероятными.

Описанная выше система полностью определяется размерностью $d$, числом $M$ и последовательностью $\left\{R_{i}\right\}$. Введем зависящие от указанных данных числовые характеристики системы, которые играют важную роль в формулировке результатов.

Определим число $b=b(M)$ как положительное решение уравнения

$$
M=\sum_{j=1}^{\infty} j\left[\frac{q_{j}}{e^{b j}-1}-\frac{q_{j}\left(R_{j}+1\right)}{e^{\left(R_{j}+1\right) b j}-1}\right] .
$$

(Здесь и в дальнейшем мы принимаем для сокращения записи соглашение, при котором подразумевается, что в такого рода суммах слагаемые, содержащие $R_{j}$, опускаются для тех $j$, для которых $R_{j}=\infty$.)

ПРЕДЛОЖЕНИЕ 6. Для любого $M>0$ уравнение (23) имеет единственное решение $b=b(M)>0$. Функиия $b(M)$ монотонно убывает и стремится $\kappa$ нулю при $M \rightarrow \infty$. Более точно, при $M \rightarrow \infty$ справедливо соотношение

$$
M \asymp b^{-d-1} \text {. }
$$

Здесь запись $A \asymp B$ означает, что частное $A / B$ ограничено сверху и снизу положительными постоянными.

ДокАЗАТЕЛЬСтво будет дано в п. 4.2.

Как мы увидим ниже, выражения в квадратных скобках в формуле (23) играют роль "предельного распределения", вблизи которого в определенном смысле концентрируются для большинства вариантов суммарные числа заполнения

$$
N_{j}=\sum_{k=1}^{q_{j}} N_{j k}
$$


соответствующих уровней $j$. Поэтому естественно ввести для этих выражений специальное обозначение. Именно, мы положим

$$
\bar{N}_{j}=q_{j}\left[\frac{1}{e^{b j}-1}-\frac{R_{j}+1}{e^{\left(R_{j}+1\right) b j}-1}\right], \quad j=1,2, \ldots,
$$

где $b$ - решение уравнения (23). Далее, обозначим через

$$
N=\sum_{j=1}^{\infty} \bar{N}_{j} \equiv \sum_{j=1}^{\infty}\left[\frac{q_{j}}{e^{b j}-1}-\frac{q_{j}\left(R_{j}+1\right)}{e^{\left(R_{j}+1\right) b j}-1}\right]
$$

сумму всех чисел (26). Число $N$, как мы опять же увидим ниже, представляет собой то значение, около которого концентрируются в большинстве вариантов сумма чисел заполнения, т.е. общее число частиц в системе.

ПреДЛОЖЕНИЕ 7. Пусть $d>1$. Тогда для величины $N$, задаваемой формулой (27), выполнены при $M \rightarrow \infty$ соотношения

$$
N \asymp b^{-d}, \quad M \asymp N^{(d+1) / d} .
$$

Eсли $d=1$ и при этом все $R_{j}$ одинаковы и конечны, $R_{j}=R<\infty$ для всех $j$, то справедливы те же соотношения. Наконеи, если $d=1$ u $R_{j}=\infty$ для всех $j$, то выполнена оценка

$$
N \asymp b^{-1}|\ln b|
$$

\section{ДОКАЗАТЕЛЬСТВО см. в П. 4.2 .}

Всюду далее (если явно не оговорено противное) считается, что $M, N$ и $b$ связаны формулами (23), (27).

Обозначим через $\mathscr{N}(M)$ общее число наборов $\left\{N_{j k}\right\}$, удовлетворяющих ограничениям (21), (22).

ОПРЕДЕЛЕНИЕ 8. Энтропией рассматриваемой системы назовем число

$$
S=\ln \mathscr{N}(M) .
$$

Асимптотику энтропии при $M \rightarrow \infty$ дает следующая теорема.

Теорема 9. При $M \rightarrow \infty$ справедлива асимптотическая формула

$$
S \equiv \ln \mathscr{N}(M)=b M+\sum_{j=1}^{\infty} q_{j} \ln \frac{1-e^{-\left(R_{j}+1\right) b j}}{1-e^{-b j}}+O(\ln N) .
$$

ДокАзАТЕЛЬСтво будет дано в п. 2.2.

1.3.2. Теоремы о концентрации. Всюду в этом пункте предполагается, что $d>2$. Доказательства теорем из этого пункта будут даны в п. 3.2.

Пусть $\psi(x)$ - фиксированнная положительная функция, которая стремится $\mathrm{K}+\infty$ при $x \rightarrow+\infty$, и пусть

$$
\Delta N=\sqrt{N \ln N} \psi(N), \quad \Delta M=\frac{M}{N} \Delta N .
$$


Теорема 10. Существуют такие постоянные $C_{k}, k=1,2, \ldots$, что

$$
\begin{gathered}
\mathrm{P}\left(\left|\sum_{j} N_{j}-N\right|>\Delta N\right) \leqslant C_{k} N^{-k}, \\
\mathrm{P}\left(\left|\sum_{j} j N_{j}-M\right|>\Delta M\right) \leqslant C_{k} N^{-k} .
\end{gathered}
$$

Для кумулятивных вероятностей справедлива следующая теорема.

Теорема 11. Существуют такие постоянные $C_{k}, k=1,2, \ldots$, что для любого $l \in \mathbb{Z}_{+}$справедливы оценки

$$
\begin{aligned}
& \mathrm{P}\left(\left|\sum_{j=1}^{l} N_{j}-\sum_{j=1}^{l} \bar{N}_{j}\right|>\Delta N\right) \leqslant C_{k} N^{-k}, \\
& \mathrm{P}\left(\left|\sum_{j=1}^{l} j N_{j}-\sum_{j=1}^{l} j \bar{N}_{j}\right|>\Delta M\right) \leqslant C_{k} N^{-k},
\end{aligned}
$$

где числа $\bar{N}_{j}$ определены в (26).

Теоремы 10 и 11 суть частные случаи следующей более общей теоремы.

ТЕОРема 12. Пусть $f=\left\{f_{j}\right\}, j=1,2, \ldots,-$ произвольная последовательность комплексных чисел, такая, что

$$
\left|f_{j}\right| \leqslant C_{0} j^{\alpha}, \quad j=1,2, \ldots,
$$

где $\alpha \in[0,1]$ - заданное число. Обозначим

$$
\Delta=N^{\alpha / d} \Delta_{N}
$$

где $\Delta_{N}=\sqrt{N \ln N} \psi(N)$, а $\psi(x)$ - функиия, сколь угодно медленно растущая на бесконечности (см. (32)).

Пусть набор $\left\{N_{j k}\right\}$ неотрицательных целых чисел случайным и равновероятным для всех вариантов образом выбирается из множества (21), (22). Определим числа $N_{j}$ второй из бормул в (22). Тогда существуют такие постоянные $C_{k}$, $k=1,2, \ldots$, зависящие только от $\alpha$ и $C_{0}$, что при всех $M$ справедливы оценки

$$
\mathrm{P}\left(\left|\sum_{j=1}^{\infty} f_{j}\left(N_{j}-\bar{N}_{j}\right)\right|>\Delta\right) \leqslant C_{k} N^{-k},
$$

где числа $\bar{N}_{j}$ определены в (26).

Теорема 12 фактически устанавливает слабую сходимость величин $N_{j}$ с вероятностью 1 к предельному распределению $\bar{N}_{j}$ при $M \rightarrow \infty$. Оказывается, однако, что справедлив и более сильный результат, который мы сформулируем для случая $R_{j}=\infty, j=1,2, \ldots$

Определим случайную функцию $\xi_{M}(x)$ на полуоси $\mathbb{R}_{+}=(0, \infty)$ следующим образом. Из множества $\Omega_{M}$ всех наборов $\left\{N_{j k}\right\}, k=1, \ldots, q_{j}$, неотрицательных целых 
чисел таких, что

$$
\begin{gathered}
N_{j k} \in\{0,1, \ldots\}, \\
\sum_{j=1}^{\infty} j \sum_{k=1}^{q_{j}} N_{j k} \leqslant M,
\end{gathered}
$$

случайным образом (считая, что все они равновероятны) выбираем какой-либо набор $\left\{N_{j k}\right\}$ и полагаем

$$
\xi_{M}(x)=b^{d-1} \sum_{k=1}^{q_{j}} N_{j k} \quad \text { при } \quad x \in[b(j-1), b j), \quad j=0,1,2, \ldots
$$

Таким образом, получаем последовательность $\xi_{M}(x), M=1,2, \ldots$, независимых случайных функций.

Определим также функцию $\xi(x)$ формулой

$$
\xi(x)=\frac{1}{\Gamma(d-1)} \frac{x^{d-1}}{e^{x}-1} .
$$

Зафиксируем неотрицательные числа $\gamma$ и $\delta$ такие, что

$$
\gamma<1-\frac{2}{d}, \quad \delta<\frac{2}{d}
$$

а также достаточно большую положительную постоянную $\mu$ (точное значение которой можно получить, если проследить за константами в доказательстве теоремы 13). Для каждого $M$ положим ${ }^{1}$

$$
\sigma=N^{-\delta}
$$

и зададим точки $x_{l} \in \mathbb{R}_{+}, l=1, \ldots, s \leqslant N^{\gamma}$, формулой

$$
x_{l}=\mu \sigma l, \quad l=1, \ldots, s .
$$

Пусть $f_{l}(x)$ - гауссовы экспоненты

$$
f_{l}(x)=e^{-\left(x-x_{l}\right)^{2} / 2 \sigma^{2}}, \quad l=1, \ldots, s,
$$

а $P_{M}$ - проектор в $L^{2}\left(\mathbb{R}_{+}\right)$на подпространство, порожденное функциями (45).

Теорема 13. С вероятностъю 1 имеет место сходимость

$$
\left\|P_{M}\left(\xi_{M}-\xi\right)\right\|_{L^{2}\left(\mathbb{R}_{+}\right)} \rightarrow 0 \quad \text { npu } \quad M \rightarrow \infty .
$$

Теорема 13 допускает эквивалентную “невероятностную” формулировку. Рассмотрим прямое произведение

$$
\Omega^{\infty}=\prod_{M=1}^{\infty} \Omega_{M}
$$

определенных выше конечных множеств $\Omega_{M}$ и снабдим его минимальной $\sigma$-алгеброй множеств, содержащей всевозможные иилиндрические множества

$$
A=A_{1} \times A_{2} \times \cdots \times A_{k} \times \Omega_{k+1} \times \Omega_{k+2} \cdots,
$$

\footnotetext{
${ }^{1}$ Напомним, что $M, N$ и $b$ связаны формулами (23) и (27).
} 
где $k$ - произвольное конечное, а $A_{j} \subset \Omega_{j}$ - произвольные подмножества. Определим на $\Omega^{\infty}$ меру, задавая ее на множествах вида (47) формулой

$$
d \mu(A)=\frac{\#\left(A_{1}\right)}{\#\left(\Omega_{1}\right)} \times \frac{\#\left(A_{2}\right)}{\#\left(\Omega_{2}\right)} \times \cdots \times \frac{\#\left(A_{k}\right)}{\#\left(\Omega_{k}\right)}
$$

и продолжая на всю $\sigma$-алгебру стандартным образом. (Здесь \# $(A)$ - число элементов в множестве $A$.)

Зададим теперь функции $\xi_{M}(x, \omega), x \in \mathbb{R}_{+}, \omega \in \Omega^{\infty}$, полагая

$$
\begin{aligned}
\xi(x, \omega) & =\frac{1}{\Gamma(d-1)} \frac{x^{d-1}}{e^{x}-1}, \\
\xi_{M}(x, \omega) & =b^{d-1} \sum_{k=1}^{q_{j}} N_{j k} \quad \text { при } \quad x \in[b(j-1), b j), \quad j=0,1,2, \ldots,
\end{aligned}
$$

если $\omega=\left(\omega_{1}, \ldots, \omega_{M}, \ldots\right)$, причем $\omega_{M}=\left\{N_{j k}\right\}$. Таким образом, $\xi$ вовсе не зависит от аргумента $\omega$, а $\xi_{M}$ зависит только от компоненты $\omega_{M}$. Так определенные функции являются элементами пространства

$$
B=L^{1}\left(\Omega^{\infty}, d \mu ; L^{2}\left(\mathbb{R}_{+}\right)\right)
$$

функций класса $L^{1}$ относительно меры $d \mu$ на пространстве $\Omega^{\infty}$ со значениями в гильбертовом пространстве $L^{2}\left(\mathbb{R}_{+}\right)$.

Определим проекторы $\mathscr{P}_{M}$ в пространстве $B$, полагая

$$
\left[\mathscr{P}_{M} u\right](\cdot, \omega)=P u(\cdot, \omega)
$$

(определенный выше проектор $P$ применяется к функции $u$, рассматриваемой как элемент пространства $L^{2}\left(\mathbb{R}_{+}\right)$при каждом фиксированном $\left.\omega\right)$.

Tеорема 14. Имеет место сходимость

$$
\left\|\mathscr{P}_{M}\left(\xi_{M}-\xi\right)\right\|_{B} \rightarrow 0 \quad \text { npu } \quad M \rightarrow \infty
$$

1.3.3. Интегральные формулы для $M$ и $N$ и поправки к ним. В формулах (27) и (23) величины $N$ и $M$ выражаются как функции от $b$ в виде бесконечных сумм. Оказывается, однако, что если $R(x) \equiv R=$ const, то эти функции можно разложить по степеням $b$ (при этом число членов разложения зависит от размерности $d$ ). Сформулируем соответствующее утверждение.

ПРеДЛОЖеНИЕ 15. Пусть $R_{j} \equiv R=$ const. Тогда при $b \rightarrow 0$ имеют место асимптотические разложения

$$
\begin{aligned}
M & =b^{-d-1}\left(K_{0}+K_{1} b+\cdots+K_{m} b^{m}+O\left(b^{m+1}\right)\right), \\
N & =b^{-d}\left(L_{0}+L_{1} b+\cdots+L_{m} b^{m}+O\left(b^{m+1}\right)\right),
\end{aligned}
$$


где $m=\lceil d / 2-1\rceil$ - наименьшее целое число, не менъшее, чем $d / 2-1$, а коэффициенты разложения выражаются явными формулами. В частности,

$$
\begin{aligned}
K_{0} & =\frac{1}{\Gamma(d)} \int_{0}^{\infty}\left[\frac{x^{d}}{e^{x}-1}-\frac{(R+1) x^{d}}{e^{x(R+1)}-1}\right] d x=\frac{\Gamma(d+1) \zeta(d+1)\left(1-(R+1)^{-d}\right)}{\Gamma(d)} \\
L_{0} & =\frac{1}{\Gamma(d)} \int_{0}^{\infty}\left[\frac{x^{d-1}}{e^{x}-1}-\frac{(R+1) x^{d-1}}{e^{x(R+1)}-1}\right] d x=\zeta(d)\left(1-(R+1)^{-d+1}\right) \\
K_{1} & =\frac{d(d-1)}{2 \Gamma(d)} \int_{0}^{\infty}\left[\frac{x^{d-1}}{e^{x}-1}-\frac{(R+1) x^{d-1}}{e^{x(R+1)}-1}\right] d x=\frac{d(d-1)\left(1-(R+1)^{-d+1}\right) \zeta(d)}{2}, \\
L_{1} & =\frac{d(d-1)}{2 \Gamma(d)} \int_{0}^{\infty}\left[\frac{x^{d-2}}{e^{x}-1}-\frac{(R+1) x^{d-2}}{e^{x(R+1)}-1}\right] d x \\
& =\frac{d(d-1) \Gamma(d-1) \zeta(d-1)}{2 \Gamma(d)}\left(1-(R+1)^{-d+2}\right)
\end{aligned}
$$

где $(s)$ - дзета-функиия Эйлера. (Из доказательства будет ясно, как в явном виде вычислять последующие члены.) Если $R=\infty$, то содержащие $R+1$ слагаемые в правых частях формул (51) и (52) следует отбросить.

ЗАмечАниЕ 16. Как показывает теорема 10, не имеет смысла получать асимптотические разложения величин $M$ и $N$ с большей точностью, чем это сделано в предложении 15. Действительно, вычислять $N$ имеет смысл с точностью до $O\left(N^{1 / 2}\right)=O\left(b^{-d / 2}\right)$, а $M-$ с точностью до $O\left((M / N) N^{1 / 2}\right)=O\left(b^{-d / 2+1}\right)$. Отсюда и получается условие на $m$.

\section{2. Общее число вариантов и энтропия}

В этом параграфе мы докажем оценки снизу и сверху для общего числа $\mathscr{N}(M)$ вариантов наборов $\left\{N_{j k}\right\}$, удовлетворяющих условиям $(21),(22)$, и выведем из этих оценок теорему 9 об энтропии.

\section{1. Оценка снизу для общего числа вариантов $\mathscr{N}(M)$.}

ПреДЛОЖеНИЕ 17. Для общего числа вариантов $\mathscr{N}(M)$ справедлива при $M \rightarrow \infty$ оченка

$$
\mathscr{N}(M) \geqslant C b^{1+d / 2} \exp \left\{b M+\sum_{j=1}^{\infty} q_{j} \ln \frac{1-e^{-\left(R_{j}+1\right) b j}}{1-e^{-b j}}\right\},
$$

где $b=b(M)$ определяется из уравнения (23).

ДокАЗАТЕЛЬСтво. Чтобы доказать оценку (57), нам будет удобно наряду с общим числом $\mathscr{N}(M)$ вариантов наборов $\left\{N_{j k}\right\}$, для которых суммарная энергия не превосходит $M$, ввести число $\mathscr{N}_{0}(M)$ наборов с энергией, в точности равной $M$, т.е. число целочисленных наборов $\left\{N_{j k}\right\}, j=1,2, \ldots, k=1, \ldots, q_{j}$, таких, что

$$
\begin{gathered}
\left.N_{j k} \in\left\{0,1, \ldots, R_{j}\right\} \quad \text { (или } N_{j k} \in\{0,1,2, \ldots\}, \text { если } R_{j}=\infty\right), \\
\sum_{j=1}^{\infty} j N_{j} \equiv \sum_{j=1}^{\infty} j \sum_{k=1}^{q_{j}} N_{j k}=M .
\end{gathered}
$$


Очевидно, что $\mathscr{N}(M) \geqslant \mathscr{N}_{0}(M)$, поэтому достаточно доказать оценку снизу для $\mathscr{N}_{0}(M)$. Дальнейшие рассуждения состоят из двух частей. Сначала мы выразим $\mathscr{N}_{0}(M)$ в виде быстроосциллирующего интеграла, а затем оценим этот интеграл с помощью рассуждений, близких к методу Лапласа.

2.1.1. Интегральная формула для $\mathscr{N}_{0}(M)$. В этом пункте мы предъявим для числа $\mathscr{N}_{0}(M)$ интегральную формулу. Для того, чтобы ее получить, представим $\mathscr{N}_{0}(M)$ в виде суммы

$$
\mathscr{N}_{0}(M)=\sum_{\left\{N_{j k}\right\}} \delta_{M, \sum_{j=1}^{\infty} j N_{j}}=\sum_{\left\{N_{j k}\right\}} \delta_{M, \sum_{j=1}^{\infty} j N_{j}} e^{b\left(M-\sum_{j=1}^{\infty} j N_{j}\right)}
$$

по всевозможным наборам $\left\{N_{j k}\right\}$ неотрицательных целых чисел, удовлетворяющих условию (58), где

$$
\delta_{m n}= \begin{cases}1, & m=n, \\ 0, & m \neq n\end{cases}
$$

- символ Кронекера. Справедливость представления (60) вытекает из того, что показатель экспоненты в (60) равен нулю для всех ненулевых членов, так что это выражение есть просто сумма единиц по всем наборам $\left\{N_{j k}\right\}$, удовлетворяющим (58), (59). Формула (60) справедлива, разумеется, для произвольного $b$. В дальнейшем в качестве $b$ мы будем использовать решение $b=b(M)>0$ уравнения (23). Подставляя в (60) представление символа Кронекера в виде

$$
\delta_{m n}=\frac{b}{2 \pi} \int_{-\pi / b}^{\pi / b} e^{i b \varphi(m-n)} d \varphi
$$

и переставляя суммирование и интегрирование (что законно, так как даже если ряд из подынтегральных выражений и бесконечен, все равно при $b>0$ он абсолютно сходится), получаем

$$
\mathscr{N}_{0}(M)=\frac{b e^{b M}}{2 \pi} \int_{-\pi / b}^{\pi / b} e^{i b \varphi M} \sum_{\left\{N_{j k}\right\}} e^{-b \sum_{j=1}^{\infty} j N_{j}(1+i \varphi)} d \varphi .
$$

Далее, разлагая экспоненту в произведение экспонент, а сумму - в произведение сумм по всем $N_{j k}$ и суммируя геометрические прогрессии, получаем

$$
\begin{aligned}
\sum_{\left\{N_{j k}\right\}} e^{-b \sum_{j=1}^{\infty} j N_{j}(1+i \varphi)} & =\sum_{\left\{N_{j k}\right\}} e^{-b \sum_{j=1}^{\infty} \sum_{k=1}^{q_{j}} j N_{j k}(1+i \varphi)} \\
& =\prod_{j=1}^{\infty}\left(\sum_{N_{j 1}=0}^{R_{j}} e^{-b j N_{j 1}(1+i \varphi)} \ldots \sum_{N_{j q_{j}}=0}^{R_{j}} e^{-b j N_{j q_{j}}(1+i \varphi)}\right) \\
& =\prod_{j=1}^{\infty}\left(\frac{1-e^{-\left(R_{j}+1\right) b j(1+i \varphi)}}{1-e^{-b j(1+i \varphi)}}\right)^{q_{j}} .
\end{aligned}
$$

Подстановка этого выражения в (61) дает

$$
\mathscr{N}_{0}(M)=\frac{b e^{b M}}{2 \pi} \int_{-\pi / b}^{\pi / b} \exp \left\{i b \varphi M+\sum_{j=1}^{\infty} q_{j} \ln \frac{1-e^{-\left(R_{j}+1\right) b j(1+i \varphi)}}{1-e^{-b j(1+i \varphi)}}\right\} d \varphi .
$$


2.1.2. Оценка снизу для $\mathscr{N}_{0}(M)$ при $M \rightarrow \infty$. Оценим теперь $\mathscr{N}_{0}(M)$ снизу, пользуясь интегральным представлением (62). Перепишем (62) в виде

$$
\mathscr{N}_{0}(M)=\frac{b e^{b M}}{2 \pi} \int_{-\pi / b}^{\pi / b} \exp \left\{b^{-d} \Phi(\varphi)\right\} d \varphi
$$

где фаза $\Phi(\varphi)$ задается формулой

$$
\Phi(\varphi)=i b^{d+1} M \varphi+b^{d} \sum_{j=1}^{\infty} q_{j} \ln \frac{1-e^{-\left(R_{j}+1\right) b j(1+i \varphi)}}{1-e^{-b j(1+i \varphi)}} .
$$

Интеграл (63) можно понимать как интеграл по окружности радиуса $1 / b$. Действительно, второе слагаемое в формуле (64) само $2 \pi / b$-периодично, а значения первого слагаемого, умноженного на $b^{-d}$, на концах отрезка интегрирования отличаются на $2 \pi M$, так что экспонента в (63) $2 \pi / b$-периодична. (Напомним, что $M$ пробегает целые положительные значения.)

Оценить интеграл (63) можно с помощью метода Лапласа [9], считая малым параметром величину $h=b^{-d}$. Отметим, что сама фазовая функция $\Phi(\varphi)$ достаточно сложным образом зависит от этого параметра. Следующая лемма по существу означает, что условия применимости метода Лапласа выполнены в рассматриваемом случае. Однако формулировку метода Лапласа в нужной форме нам в литературе обнаружить не удалось, и поэтому далее приходится непосредственно провести оценку интеграла, используя полученные в лемме утверждения. Рассуждения при этом оказываются несколько проще, чем они были бы в доказательстве метода Лапласа, поскольку нас интересует не асимптотика, а лишь оценка снизу. (Подчеркнем, что никаких принципиальных препятствий к получению асимптотики нет.)

ЛЕмма 18. Задаваемая формулой (64) фазовая функиия $\Phi(\varphi)$ обладает следующими свойствами.

1) Все производные фазовой функиии (64) ограничены равномерно по $M \rightarrow \infty$ постоянными, не зависящими от выбора последовательности $\left\{R_{j}\right\}$.

2) Фазовая функиия имеет при каждом $M$ невырожденную стационарную точ$\kappa y \varphi=0 \bmod 2 \pi / b$.

3) В точке $\varphi=0$ мнимая часть функиии $\Phi(\varphi)$ равна нулю, а ее вещественная часть достигает абсолютного максимума, причем для любого $\varepsilon>0$ существует такое $\delta>0$, не зависящее от числа $M$ и последовательности $\left\{R_{j}\right\}$, чmo

$$
\operatorname{Re} \Phi(\varphi)<\operatorname{Re} \Phi(0)-\delta \quad n p u \quad \varphi \in[-\pi / b,-\varepsilon) \cup(\varepsilon, \pi / b] .
$$

4) Вторая производная $\Phi^{\prime \prime}(0)$ вещественна и удовлетворяет оценке

$$
\Phi^{\prime \prime}(0)<-C<0,
$$

где постоянная $C$ не зависит от $M$ и последовательности $\left\{R_{j}\right\}$.

Доказательство. 1. Докажем, что производные функции $\Phi(\varphi)$ равномерно ограничены. Для этого вычислим их. Начнем с первой производной:

$$
\Phi^{\prime}(\varphi)=i b^{d+1} M+i b^{d+1} \sum_{j=1}^{\infty} j q_{j}\left[\frac{R_{j}+1}{e^{\left(R_{j}+1\right) b j(1+i \varphi)}-1}-\frac{1}{e^{b j(1+i \varphi)}-1}\right]
$$


Первое слагаемое здесь ограничено, поскольку $M \asymp b^{-d-1}$ в силу предложения 6 . Чтобы оценить второе слагаемое, заметим, что

$$
\begin{aligned}
\left|\frac{1}{e^{b j(1+i \varphi)}-1}\right| & =\left|\frac{1}{e^{b j}(\cos b j \varphi+i \sin b j \varphi)-1}\right| \\
& =\frac{1}{\sqrt{\left(e^{b j} \cos b j \varphi-1\right)^{2}+\left(e^{b j} \sin b j \varphi\right)^{2}}} \\
& =\frac{1}{\sqrt{\left(e^{b j}-1\right)^{2}+2 e^{b j}(1-\cos b j \varphi)}} \leqslant \frac{1}{e^{b j}-1}
\end{aligned}
$$

(причем равенство достигается только тогда, когда $b j \varphi=0 \bmod 2 \pi$ ). Аналогично,

$$
\left|\frac{R_{j}+1}{e^{\left(R_{j}+1\right) b j(1+i \varphi)}-1}\right| \leqslant \frac{R_{j}+1}{e^{\left(R_{j}+1\right) b j}-1}<\frac{1}{e^{b j}-1} ;
$$

второе неравенство в (69) верно, поскольку

$$
\frac{1}{e^{b j}-1}-\frac{R_{j}+1}{e^{\left(R_{j}+1\right) b j}-1}=\frac{1}{e^{b j}-1}[1-\underbrace{\frac{R_{j}+1}{1+e^{b j}+e^{2 b j}+\cdots+e^{R_{j} b j}}}_{R_{j}+1 \text { слагаемых }}]>0 .
$$

Далее, по формуле Стирлинга

$$
q_{j} \simeq \frac{j^{d-1}}{\Gamma(d)}
$$

Применяя лемму 23 (при $l=d \geqslant 1, m=1$ и $k=0$ ), убеждаемся, что сумма в (67) имеет порядок не выше $b^{-d-1}$, так что и второе слагаемое в формуле для $\Phi^{\prime}(\varphi)$ ограничено. Ограниченность первой производной доказана.

Вычислим последующие производные, начиная со второй. Имеем

$$
\Phi^{\prime \prime}(\varphi)=b^{d+2} \sum_{j=1}^{\infty} q_{j}\left[\frac{\left(R_{j}+1\right)^{2} j^{2} e^{\left(R_{j}+1\right) b j(1+i \varphi)}}{\left(e^{\left(R_{j}+1\right) b j(1+i \varphi)}-1\right)^{2}}-\frac{j^{2} e^{b j(1+i \varphi)}}{\left(e^{b j(1+i \varphi)}-1\right)^{2}}\right],
$$

а при $s \geqslant 2$

$$
\Phi^{(s)}(\varphi)=b^{d+s} \sum_{2 \leqslant m \leqslant s} C_{s k m} \sum_{j=1}^{\infty} q_{j}\left[\frac{\left(R_{j}+1\right)^{s} j^{s} e^{(m-1)\left(R_{j}+1\right) b j(1+i \varphi)}}{\left(e^{\left(R_{j}+1\right) b j(1+i \varphi)}-1\right)^{m}}-\frac{j^{s} e^{(m-1) b j(1+i \varphi)}}{\left(e^{b j(1+i \varphi)}-1\right)^{m}}\right],
$$

где $C_{s k m}$ - некоторые постоянные. Действительно, (71) получается из (67) непосредственным дифференцированием, а (72) согласуется с (71) при $s=2$, так что остается лишь проверить индуктивный шаг $s \rightarrow s+1$, для чего достаточно продифференцировать отдельное слагаемое в правой части (72). Например,

$$
\left[\frac{e^{k b j(1+i \varphi)}}{\left(e^{b j(1+i \varphi)}-1\right)^{m}}\right]^{\prime}=\frac{i b j e^{k b j(1+i \varphi)}}{\left(e^{b j(1+i \varphi)}-1\right)^{m}}-\frac{i m b j e^{(k+1) b j(1+i \varphi)}}{\left(e^{b j(1+i \varphi)}-1\right)^{m+1}},
$$

и аналогично для слагаемого, содержащего $R_{j}+1$.

Теперь, пользуясь оценками (68) и (69), асимптотикой (70) и леммами 23 и 24, получаем равномерную по $M \rightarrow \infty$ и последовательностям $\left\{R_{j}\right\}$ ограниченность производной $\Phi^{(s)}(\varphi)$ для любого $s$. 
2. Покажем, что $\varphi=0$ - стационарная точка фазовой функции. Действительно, при $\varphi=0$ правая часть в (67) равна нулю в силу уравнения (23). Невырожденность доказана ниже в п. 4 .

3. Из формулы (64) вытекает, что

$$
\operatorname{Re} \Phi(\varphi)=b^{d} \sum_{j=1}^{\infty} q_{j} \ln \left|1+e^{-b j(1+i \varphi)}+e^{-2 b j(1+i \varphi)}+\cdots+e^{-R_{j} b j(1+i \varphi)}\right|
$$

(при $R_{j}=\infty$ ряд под знаком логарифма будет бесконечный) и соответственно

$$
\begin{aligned}
& \operatorname{Re} \Phi(0)-\operatorname{Re} \Phi(\varphi)= b^{d} \sum_{j=1}^{\infty} q_{j}\left[\ln \left(1+e^{-b j}+e^{-2 b j}+\cdots+e^{-R_{j} b j}\right)\right. \\
&\left.\quad-\ln \left|1+e^{-b j(1+i \varphi)}+e^{-2 b j(1+i \varphi)}+\cdots+e^{-R_{j} b j(1+i \varphi)}\right|\right] \\
& \equiv b^{d} \sum_{j=1}^{\infty} q_{j} \Delta_{j} .
\end{aligned}
$$

Оценим отдельные слагаемые в сумме (73). Справедливо неравенство

$$
\begin{aligned}
\mid 1+e^{-b j(1+i \varphi)} & +e^{-2 b j(1+i \varphi)}+\cdots+e^{-R_{j} b j(1+i \varphi)} \mid \\
& \leqslant\left|1+e^{-b j(1+i \varphi)}\right|+e^{-2 b j}+\cdots+e^{-R_{j} b j},
\end{aligned}
$$

и поэтому, в силу монотонного возрастания логарифма,

$$
\begin{aligned}
\Delta_{j} \geqslant & \ln \left(1+e^{-b j}+e^{-2 b j}+\cdots+e^{-R_{j} b j}\right) \\
& \quad-\ln \left(\left|1+e^{-b j(1+i \varphi)}\right|+e^{-2 b j}+\cdots+e^{-R_{j} b j}\right) \\
\geqslant & \frac{1+e^{-b j}-\left|1+e^{-b j(1+i \varphi)}\right|}{1+e^{-b j}+e^{-2 b j}+\cdots+e^{-R_{j} b j}} \\
\geqslant & \left(1+e^{-b j}-\left|1+e^{-b j(1+i \varphi)}\right|\right)\left(1-e^{-b j}\right)
\end{aligned}
$$

(при переходе к предпоследней строчке мы воспользовались очевидным неравенством $\ln y-\ln x \geqslant(y-x) / y$ при $y>x>0)$.

Далее,

$$
\begin{aligned}
1+e^{-b j}-\mid 1+e^{-b j(1+i \varphi) \mid} & =1+e^{-b j}-\sqrt{\left(1+e^{-b j} \cos b j \varphi\right)^{2}+e^{-2 b j} \sin ^{2} b j \varphi} \\
& =1+e^{-b j}-\sqrt{\left(1+e^{-b j}\right)^{2}-2 e^{-b j}(1-\cos b j \varphi)} \\
& =\left(1+e^{-b j}\right)\left[1-\sqrt{1-\frac{2 e^{-b j}}{\left(1+e^{-b j}\right)^{2}}(1-\cos b j \varphi)}\right] \\
& \geqslant \frac{e^{-b j}}{1+e^{-b j}}(1-\cos b j \varphi) \geqslant \frac{e^{-b j}}{2}(1-\cos b j \varphi)
\end{aligned}
$$

(при переходе к последней строчке мы воспользовались неравенством $1-\sqrt{x} \geqslant$ $(1-x) / 2$ при $x \in[0,1])$. Подставляя это в $(74)$, получаем

$$
\Delta_{j} \geqslant \frac{1}{2} e^{-b j}\left(1-e^{-b j}\right)(1-\cos b j \varphi) .
$$


Итак, все $\Delta_{j}$ неотрицательны, а это значит, что $\operatorname{Re} \Phi(\varphi)$ действительно достигает абсолютного максимума при $\varphi=0$.

Покажем теперь, что неравенство (65) выполняется для любого $\varepsilon>0$ при подходящим образом подобранном $\delta>0$. Зафиксируем не зависящие от $b$ числа $x_{1}, x_{2} \in$ $(0, \infty), x_{2}>x_{1}$; тогда

$$
\begin{aligned}
\operatorname{Re} \Phi(0) & -\operatorname{Re} \Phi(\varphi) \\
& =b^{d} \sum_{j=1}^{\infty} q_{j} \Delta_{j} \\
& \geqslant b^{d} \sum_{b j \in\left[x_{1}, x_{2}\right]} q_{j} \Delta_{j} \\
& \geqslant K_{1} b \sum_{b j \in\left[x_{1}, x_{2}\right]}(b j)^{d-1} e^{-b j}\left(1-e^{-b j}\right)(1-\cos b j \varphi) \\
& \geqslant K_{2} b \sum_{b j \in\left[x_{1}, x_{2}\right]}(1-\cos b j \varphi),
\end{aligned}
$$

где постоянные $K_{1}, K_{2}>0$ зависят только от $x_{1}, x_{2}$.

Пусть $j_{1}=j_{1}(b), j_{2}=j_{2}(b)$ - минимальное и максимальное целые значения $j$, при которых $b j \in\left[x_{1}, x_{2}\right]$. Тогда

$$
j_{2}-j_{1} \geqslant \frac{x_{2}-x_{1}}{b}-2 .
$$

Согласно [10; 1.341.3],

$$
\begin{aligned}
b \sum_{b j \in\left[x_{1}, x_{2}\right]}(1-\cos b j \varphi) & =b \sum_{j=j_{1}}^{j_{2}}(1-\cos (j b \varphi)) \\
& =b\left[j_{2}-j_{1}+1+\frac{\cos \left(\left(j_{1}+j_{2}\right) b \varphi / 2\right) \sin \left(\left(j_{2}-j_{1}+1\right) b \varphi / 2\right)}{\sin (b \varphi / 2)}\right] \\
& \geqslant x_{2}-x_{1}-b-\left|\sin \frac{b \varphi}{2}\right|^{-1} .
\end{aligned}
$$

Пусть $\varphi \in[-\pi / b,-\varepsilon] \cup[\varepsilon, \pi / b]$; в силу нечетности синуса достаточно рассмотреть случай, когда $\varphi>0$. Тогда $b \varphi / 2 \in[b \varepsilon / 2, \pi / 2]$, так что

$$
\sin \frac{b \varphi}{2} \geqslant \sin \frac{b \varepsilon}{2} \geqslant \frac{2}{\pi} \frac{b \varepsilon}{2}=\frac{b \varepsilon}{\pi}, \quad \sin ^{-1} \frac{b \varphi}{2} \leqslant \frac{\pi}{b \varepsilon},
$$

и, комбинируя это с предыдущими неравенствами, получаем, равномерно по $b$ при $b \rightarrow 0$,

$$
\operatorname{Re} \Phi(0)-\operatorname{Re} \Phi(\varphi) \geqslant K_{2}\left[x_{2}-x_{1}-b-\frac{\pi}{\varepsilon}\right] .
$$

(напомним, что положительная постоянная $K_{2}$ зависит от $x_{1}, x_{2}$ ). Теперь для завершения доказательства п. 3 достаточно выбрать $x_{2}>x_{1}+\pi / \varepsilon+1$.

4. Полагая $\varphi=0$ в формуле (71), получаем

$$
\Phi^{\prime \prime}(0)=-b^{d+2} \sum_{j=1}^{\infty} q_{j} j^{2}\left[\frac{e^{b j}}{\left(e^{b j}-1\right)^{2}}-\frac{\left(R_{j}+1\right)^{2} e^{\left(R_{j}+1\right) b j}}{\left(e^{\left(R_{j}+1\right) b j}-1\right)^{2}}\right] .
$$


Рассмотрим функцию

$$
f(a)=\frac{a^{2} e^{a x}}{\left(e^{a x}-1\right)^{2}},
$$

где $x>0$ произвольное фиксированное число.

УтвеРЖДЕНИЕ 19. Функция $f(a)$ монотонно убъвает при $а>0$.

ДоказАтельство. Достаточно показать, что $f^{\prime}(a)<0$ при $a>0$. Непосредственное вычисление дает

$$
f^{\prime}(a)=\frac{a e^{a x}}{\left(e^{a x}-1\right)^{3}}\left[2 e^{a x}-a x e^{a x}-a x-2\right],
$$

так что достаточно показать, что выражение в квадратных скобках отрицательно, т.е.

$$
\psi(z) \equiv 2 e^{z}-z e^{z}-z-2<0 \quad \text { при } \quad z>0 .
$$

Это действительно так, поскольку

$$
\psi(0)=0, \quad \psi^{\prime}(z)=e^{z}-z e^{z}-1=\sum_{j=2}^{\infty} z^{j}\left(\frac{1}{j !}-\frac{1}{(j-1) !}\right),
$$

и все коэффициенты последнего ряда отрицательны, что и доказывает утверждение 19.

Вернемся к доказательству утверждения 4 леммы 18. Пользуясь утверждением 19, из (76) заключаем, поскольку $R_{j} \geqslant 1$, что

$$
\Phi^{\prime \prime}(0) \leqslant-b^{d+2} \sum_{j=1}^{\infty} q_{j} j^{2}\left[\frac{e^{b j}}{\left(e^{b j}-1\right)^{2}}-\frac{4 e^{2 b j}}{\left(e^{2 b j}-1\right)^{2}}\right]=-b^{d+2} \sum_{j=1}^{\infty} q_{j} j^{2} \frac{e^{b j}}{\left(e^{b j}+1\right)^{2}} .
$$

Остается воспользоваться асимптотикой (70) кратностей $q_{j}$ и применить лемму 23.

Лемма 18 доказана.

Продолжим доказательство предложения 17.

Теперь мы можем оценить интеграл (63). По лемме 18 все производные функции $\Phi(\varphi)$ равномерно ограничены. Кроме того, если представить ее в виде $\Phi=\Phi_{1}+i \Phi_{2}$, где $\Phi_{1}$ и $\Phi_{2}$ вещественны, то

$$
\Phi_{1}^{\prime}(0)=0, \quad \Phi_{1}^{\prime \prime}(0)<-C<0, \quad \Phi_{2}^{\prime}(0)=\Phi_{2}^{\prime \prime}(0)=0 .
$$

Отсюда с помощью формулы Тейлора с остаточным членом получаем при $|\varphi| \leqslant \varepsilon$, где $\varepsilon>0$ достаточно мало, оценки

$$
\begin{gathered}
\Phi(0)-C_{1}|\varphi|^{2} \leqslant \Phi_{1}(\varphi) \leqslant \Phi(0)-C_{2}|\varphi|^{2}, \\
\left|\Phi_{2}(\varphi)\right| \leqslant C_{3}|\varphi|^{3}
\end{gathered}
$$

с положительными постоянными $C_{j}$, не зависящими от $M$ и последовательности $\left\{R_{j}\right\}$. Пусть

$$
1=\psi_{1}(\varphi)+\psi_{2}(\varphi)
$$


- неотрицательное гладкое разбиение единицы на окружности $S^{1} \ni \varphi$ радиуса $b$ такое, что $\operatorname{supp} \psi_{1} \subset[-\varepsilon, \varepsilon]$ и $\psi_{1}(\varphi)=1$ при $\varphi \in[-\varepsilon / 2, \varepsilon / 2]$. Представим интеграл

$$
I=\int_{S^{1}} \exp \left\{b^{-d} \Phi(\varphi)\right\} d \varphi
$$

в виде суммы

$$
I=\int_{S^{1}} \exp \left\{b^{-d} \Phi(\varphi)\right\} \psi_{1}(\varphi) d \varphi+\int_{S^{1}} \exp \left\{b^{-d} \Phi(\varphi)\right\} \psi_{2}(\varphi) d \varphi \equiv I_{1}+I_{2} .
$$

По лемме 18, п. 3

$$
\operatorname{Re} \Phi(\varphi) \leqslant \Phi(0)-\delta, \quad \delta>0,
$$

на носителе подынтегрального выражения в $I_{2}$, а мера носителя имеет порядок $b^{-1}$. Поэтому

$$
\left|I_{2}\right| \leqslant K \exp \left\{-b^{-d} \delta / 2+b^{-d} \Phi(0)\right\}, \quad b \rightarrow 0,
$$

с некоторой постоянной $K$. Оценим теперь интеграл $I_{1}$. Для удобства обозначим временно через $h=b^{d}$ малый параметр, который стоит в экспоненте нашего интеграла. В отрезке $D=[-\varepsilon, \varepsilon]$ выделим два подотрезка $D_{1 / 2} \subset D_{1 / 3} \subset D$, полагая

$$
D_{1 / 2}=\left[-\varepsilon h^{1 / 2}, \varepsilon h^{1 / 2}\right], \quad D_{1 / 3}=\left[-\varepsilon h^{1 / 3}, \varepsilon h^{1 / 3}\right] .
$$

Тогда

$$
\left|\frac{\Phi_{2}}{h}\right| \leqslant C_{3} \varepsilon^{3} \quad \text { при } \quad \varphi \in D_{1 / 3},
$$

так что (при достаточно малом $\varepsilon$ ) на отрезке $D_{1 / 3}$ мнимая часть аргумента экспоненты мала и справедливо соотношение

$$
\operatorname{Re} e^{\Phi(\varphi) / h} \geqslant \frac{1}{2} e^{\Phi_{1}(\varphi) / h}, \quad \varphi \in D_{1 / 3} .
$$

Далее,

$$
\frac{\Phi(0)}{h} \geqslant \frac{\Phi_{1}(\varphi)}{h} \geqslant \frac{\Phi(0)}{h}-C_{1} \varepsilon^{2}, \quad \varphi \in D_{1 / 2} .
$$

Комбинируя это с предыдущим неравенством и учитывая, что длина интервала $D_{1 / 2}$ равна $2 \varepsilon h^{1 / 2}$, получаем

$$
\operatorname{Re} \int_{D_{1 / 2}} e^{\Phi(\varphi) / h} \psi_{1}(\varphi) d \varphi \geqslant C_{4} e^{\Phi(0) / h} h^{1 / 2} .
$$

Далее,

$$
\operatorname{Re} \int_{D_{1 / 3} \backslash D_{1 / 2}} e^{\Phi(\varphi) / h} \psi_{1}(\varphi) d \varphi \geqslant 0
$$

в силу (83). Кроме этого, справедливо неравенство

$$
\frac{\Phi_{1}(\varphi)}{h} \leqslant \frac{\Phi_{1}(0)}{h}-C_{2} \varepsilon^{2} h^{-1 / 3}, \quad \varphi \in D \backslash D_{1 / 3},
$$

так что

$$
\left|\int_{D \backslash D_{1 / 3}} e^{\Phi(\varphi) / h} \psi_{1}(\varphi) d \varphi\right| \leqslant C_{5} e^{\Phi(0) / h} e^{-C_{2} \varepsilon^{2} h^{-1 / 3}} .
$$


Комбинируя все предыдущие оценки, получаем

$$
I=\operatorname{Re} I \geqslant C_{6} b^{d / 2} \exp \left\{b^{-d} \Phi(0)\right\} .
$$

Остается подставить эту оценку в формулу (63) для $\mathscr{N}_{0}(M)$ с учетом формулы (64) для фазовой функции, и в силу неравенства $\mathscr{N}(M)>\mathscr{N}_{0}(M)$ мы приходим к оценке $(57)$ для $\mathscr{N}(M)$. Предложение 17 доказано.

2.2. Оценка сверху для общего числа вариантов $\mathscr{N}(M)$ и формула для энтропии. Верхнюю оценку для общего числа вариантов получить оказывается гораздо проще, чем нижнюю. Здесь мы, по существу, используем так называемую статистическую сумму (см., например, [6]). В силу условия (22) на множестве всех наборов $\left\{N_{j k}\right\}$, удовлетворяющих условиям (21), (22), при $b>0$ справедливо неравенство

$$
\exp \left\{b M-b \sum_{j=1}^{\infty} \sum_{k=1}^{q_{j}} j N_{j k}\right\} \geqslant 1 .
$$

Поэтому

$$
\begin{aligned}
\mathscr{N}(M) & =\sum_{\left\{N_{j k}\right\}} 1 \\
& \leqslant \sum_{\left\{N_{j k}\right\}} \exp \left\{b M-b \sum_{j=1}^{\infty} \sum_{k=1}^{q_{j}} j N_{j k}\right\} \\
& \leqslant \sum_{\left\{N_{j k}\right\}} \exp \left\{b M-b \sum_{j=1}^{\infty} \sum_{k=1}^{q_{j}} j N_{j k}\right\} \\
& =e^{b M} \prod_{j=1}^{\infty} \prod_{k=1}^{q_{j}}\left[\sum_{N_{j k}=0}^{R_{j}} e^{-N_{j k} b j}\right]
\end{aligned}
$$

(а теперь просуммируем геометрические прогрессии)

$$
=\exp \left\{b M+\sum_{j=1}^{\infty} q_{j} \ln \frac{1-e^{-\left(R_{j}+1\right) b j}}{1-e^{-b j}}\right\} .
$$

Таким образом, мы доказали искомую оценку сверху. Именно, справедливо следующее утверждение.

ПрЕДЛОЖЕНИЕ 20. Для общего числа вариантов $\mathscr{N}(M)$ выполнена ощенка

$$
\mathscr{N}(M) \leqslant \exp \left\{b M+\sum_{j=1}^{\infty} q_{j} \ln \frac{1-e^{-\left(R_{j}+1\right) b j}}{1-e^{-b j}}\right\},
$$

где $b=b(M)$ - решение уравнения (23).

ЗАмечание 21. Конечно, оценка (85) выполнена при любом $b>0$, а не только при $b=b(M)$. Но оценка с $b=b(M)$ наилучшая. Это можно проверить, минимизируя правую часть в (85) по $b$ : уравнение экстремума в точности совпадает с (23), а анализируя знак второй производной фазы экспоненты, можно установить, что эта точка есть точка минимума. 
Теперь мы можем доказать теорему 9 об энтропии.

ДОКАЗАТЕЛЬСТвО ТЕОРЕМЫ 9. Комбинируя оценки из предложений 17 и 20, мы видим, что

$$
\begin{aligned}
& C b^{1+d / 2} \exp \left\{b M+\sum_{j=1}^{\infty} q_{j} \ln \frac{1-e^{-\left(R_{j}+1\right) b j}}{1-e^{-b j}}\right\} \\
& \leqslant \mathscr{N}(M) \leqslant \exp \left\{b M+\sum_{j=1}^{\infty} q_{j} \ln \frac{1-e^{-\left(R_{j}+1\right) b j}}{1-e^{-b j}}\right\} .
\end{aligned}
$$

Переходя к логарифмам, для энтропии $S=\ln \mathscr{N}(M)$ получаем

$$
b M+\sum_{j=1}^{\infty} q_{j} \ln \frac{1-e^{-\left(R_{j}+1\right) b j}}{1-e^{-b j}}-C_{1}|\ln b| \leqslant S \leqslant b M+\sum_{j=1}^{\infty} q_{j} \ln \frac{1-e^{-\left(R_{j}+1\right) b j}}{1-e^{-b j}}
$$

откуда уже непосредственно следует асимптотика (31) с учетом того факта, что если $d>1$, то $N \simeq b^{-d}$ и, следовательно, $|\ln b| \asymp \ln N$, а если $d=1$, то

$$
N \asymp b^{-1}|\ln b|, \quad \ln N \asymp|\ln b|+\ln |\ln b|=|\ln b|(1+o(1)) \simeq|\ln b| .
$$

\section{3. Концентрация вариантов вблизи предельного распределения}

3.1. Общая теорема о сходимости. Все основные результаты данной работы, касающиеся концентрации (в том или ином смысле) большинства вариантов вблизи предельного распределения (26) являются следствиями одной общей теоремы. В данном пункте мы ее сформулируем и докажем, а в следующем - покажем, как из нее выводятся теоремы о концентрации, сформулированные в п. 1.3. В этом пункте считаем, что $d>2$.

Зафиксируем функцию $\psi(x), x \in \mathbb{R}_{+}$, сколь угодно медленно растущую на бесконечности (см. (32)).

Пусть $\gamma \in[0,1), \alpha \in[0,1]$ - заданные числа, причем

$$
\frac{1-\alpha}{d}<\frac{1-\gamma}{2}
$$

Обозначим

$$
\Delta=N^{(1+\gamma) / 2+\alpha / d} \sqrt{\ln N} \psi(N) .
$$

Далее, пусть $C_{0}>0$ - заданная постоянная. Пусть набор $\left\{N_{j k}\right\}$ неотрицательных целых чисел случайным и равновероятным для всех вариантов образом выбирается из множества (21), (22). Определим числа $N_{j}$ второй из формул в $(22)$.

Тогда справедливо следующее утверждение.

ТеОрема 22. Существуют такие постоянные $C_{k}, k=1,2, \ldots$, зависящие только от $\gamma, \alpha$ и $C_{0}$, что для произвольных м числовых последовательностей $\left\{f_{j}^{(1)}\right\}, \ldots$, $\left\{f_{j}^{(s)}\right\}, j=1,2, \ldots$, где $s \leqslant N^{\gamma}$, удовлетворяющих условию

$$
\sum_{l=1}^{s}\left|f_{j}^{(l)}\right| \leqslant C_{0} j^{\alpha}, \quad j=1,2, \ldots
$$


при всех М справедливь оценки

$$
\mathrm{P}\left(\sum_{l=1}^{s}\left|\sum_{j=1}^{\infty} f_{j}^{(l)}\left(N_{j}-\bar{N}_{j}\right)\right|>\Delta\right) \leqslant C_{k} N^{-k}
$$

где числа $\bar{N}_{j}$ определенъ формулой (26).

ДокАзАтЕльство. Найдем оценку сверху для числа $\mathscr{N}(\Delta)$ наборов $\left\{N_{j k}\right\}$, удовлетворяющих условиям (21), (22), для которых, кроме того,

$$
\sum_{l=1}^{s}\left|\sum_{j=1}^{\infty} f_{j}^{(l)}\left(N_{j}-\bar{N}_{j}\right)\right|>\Delta
$$

Условие (90) можно переписать в виде

$$
\sum_{l=1}^{s}\left|\sum_{j=1}^{\infty} \sum_{k=1}^{q_{j}} f_{j}^{(l)}\left(N_{j k}-\bar{N}_{j k}\right)\right|>\Delta
$$

где

$$
\bar{N}_{j k}=\frac{\bar{N}_{j}}{q_{j}}, \quad k=1, \ldots q_{j} .
$$

Без ограничения общности можно считать, что все $f_{j}^{(l)}$ вещественны. Тогда на множестве $\mathscr{A}$ вариантов, удовлетворяющих (21), (22) и (90), выполнены неравенства

$$
\begin{gathered}
M-\sum_{j=1}^{\infty} \lambda_{j} \sum_{k=1}^{q_{j}} N_{j k} \geqslant 0 \\
\Delta-\sum_{j=1}^{\infty} \sum_{k=1}^{q_{j}}\left(\sum_{l=1}^{s}(-1)^{\sigma_{l}} f_{j}^{(l)}\right)\left(N_{j k}-\bar{N}_{j k}\right) \leqslant 0
\end{gathered}
$$

для некоторого (зависящего от $\left.\left\{N_{j k}\right\}\right)$ набора

$$
\sigma=\left(\sigma_{1}, \ldots, \sigma_{s}\right) \in\{0,1\}^{s} .
$$

Пусть $c$ - произвольное положительное число. Из неравенств (92), (93) вытекает, что на каждом из элементов множества $\mathscr{A}$ справедливо одно из $2^{s}$ неравенств

$$
b\left(M-\sum_{j=1}^{\infty} \sum_{k=1}^{q_{j}} j N_{j k}\right)-c \Delta+c \sum_{j=1}^{\infty} \sum_{k=1}^{q_{j}}\left(\sum_{l=1}^{s}(-1)^{\sigma_{l}} f_{j}^{(l)}\right)\left(N_{j k}-\bar{N}_{j k}\right) \geqslant 0 .
$$

Отсюда следует, что

$$
\begin{aligned}
e^{b M-c \Delta} e^{-b \sum_{j=1}^{\infty} \sum_{k=1}^{q_{j}} j N_{j k}} \sum_{\sigma} \exp \left\{-c \sum_{j=1}^{\infty} \sum_{k=1}^{q_{j}}\left(\sum_{l=1}^{s}(-1)^{\sigma_{l}} f_{j}^{(l)}\right) \bar{N}_{j k}\right. & \\
& \left.+c \sum_{j=1}^{\infty} \sum_{k=1}^{q_{j}}\left(\sum_{l=1}^{s}(-1)^{\sigma_{l}} f_{j}^{(l)}\right) N_{j k}\right\} \geqslant 1
\end{aligned}
$$


на множестве $\mathscr{A}$. Далее,

$$
\mathscr{N}(\Delta)=\sum_{\left\{N_{j k}\right\} \in \mathscr{A}} 1
$$

Оценим $\mathscr{N}(\Delta)$ сверху, распространяя сумму (95) на все наборы, удовлетворяющие условию (21) и заменяя при этом единицы на левую часть неравенства (94). Это можно сделать, поскольку левая часть в (94) всюду положительна, а при $\left\{N_{j k}\right\} \in \mathscr{A}$ не меньше единицы в силу (94). Разлагая экспоненту от суммы в произведение экспонент, получим тогда

$\mathscr{N}(\Delta) \leqslant e^{b M-c \Delta} \sum_{\sigma} \prod_{j=1}^{\infty} \prod_{k=1}^{q_{j}}\left[e^{-c\left(\sum_{l=1}^{s}(-1)^{\sigma} l f_{j}^{(l)}\right) \bar{N}_{j k}} \sum_{N_{j k}=1}^{R_{j}} e^{-N_{j k}\left(b j-c\left(\sum_{l=1}^{s}(-1)^{\sigma_{l}} f_{j}^{(l)}\right)\right)}\right]$

(суммируя геометрические прогрессии)

$$
\begin{gathered}
=e^{b M-c \Delta} \sum_{\sigma} \exp \left[\sum _ { j = 1 } ^ { \infty } q _ { j } \left(-c\left(\sum_{l=1}^{s}(-1)^{\sigma_{l}} f_{j}^{(l)}\right) \bar{N}_{j k}\right.\right. \\
\left.\left.+\ln \frac{1-e^{-\left(R_{j}+1\right)\left(b j-c\left(\sum_{l=1}^{s}(-1)^{\sigma} f_{j}^{(l)}\right)\right)}}{1-e^{-\left(b j-c\left(\sum_{l=1}^{s}(-1)^{\sigma} l f_{j}^{(l)}\right)\right)}}\right)\right] .
\end{gathered}
$$

Разумеется, эти выкладки справедливы в том случае, если встречающиеся в них бесконечные суммы и произведения сходятся. Для того, чтобы это так и было, возьмем число $c$ в интервале

$$
0<c<\frac{b}{2 C_{0}}
$$

Тогда в силу оценки (88) и условия $\alpha \leqslant 1$ имеем

$$
\left|c\left(\sum_{l=1}^{s}(-1)^{\sigma_{l}} f_{j}^{(l)}\right)\right| \leqslant \frac{b}{2 C_{0}} C_{0} j^{\alpha} \leqslant \frac{b j^{\alpha}}{2} \leqslant \frac{b j}{2}
$$

а значит,

$$
b j-c\left(\sum_{l=1}^{s}(-1)^{\sigma_{l}} f_{j}^{(l)}\right) \geqslant \frac{b j}{2}
$$

так что суммы геометрических прогрессий сходятся и в том случае, когда $R_{j}=\infty$. Далее, преобразуем входящие в (96) выражения

$$
\phi_{j k} \equiv \ln \frac{1-e^{-\left(R_{j}+1\right)\left(b j-c\left(\sum_{l=1}^{s}(-1)^{\sigma} l f_{j}^{(l)}\right)\right)}}{1-e^{-\left(b j-c\left(\sum_{l=1}^{s}(-1)^{\sigma_{l}} f_{j}^{(l)}\right)\right)}}-c\left(\sum_{l=1}^{s}(-1)^{\sigma_{l}} f_{j}^{(l)}\right) \bar{N}_{j k},
$$

разлагая функцию

$$
F(x)=\ln \frac{1-e^{-\left(R_{j}+1\right) x}}{1-e^{-x}}
$$


по формуле Тейлора с остаточным членом второго порядка в точке $x=b j$ :

$$
\begin{aligned}
F\left(b j-c\left(\sum_{l=1}^{s}(-1)^{\sigma_{l}} f_{j}^{(l)}\right)\right) \\
=F(b j)-F^{\prime}(b j) c\left(\sum_{l=1}^{s}(-1)^{\sigma_{l}} f_{j}^{(l)}\right) \\
\quad+\frac{1}{2} F^{\prime \prime}\left(b j-\theta c\left(\sum_{l=1}^{s}(-1)^{\sigma_{l}} f_{j}^{(l)}\right)\right)\left(c\left(\sum_{l=1}^{s}(-1)^{\sigma_{l}} f_{j}^{(l)}\right)\right)^{2},
\end{aligned}
$$

где число $\theta \in[0,1]$, разумеется, свое для каждого из выражений (100). Имеем

$$
F^{\prime}(x)=\frac{\left(R_{j}+1\right) e^{-\left(R_{j}+1\right) x}}{1-e^{-\left(R_{j}+1\right) x}}-\frac{e^{-x}}{1-e^{-x}}=\frac{R_{j}+1}{e^{\left(R_{j}+1\right) x}-1}-\frac{1}{e^{x}-1},
$$

так что с учетом формулы (26)

$$
F^{\prime}(b j)=-\bar{N}_{j k}
$$

Подставляя полученное выражение в (100), получаем

$$
\begin{aligned}
\phi_{j k}=\ln & \frac{1-e^{-\left(R_{j}+1\right) b j}}{1-e^{-b j}} \\
& +\frac{1}{2} F^{\prime \prime}\left(b j-\theta c\left(\sum_{l=1}^{s}(-1)^{\sigma_{l}} f_{j}^{(l)}\right)\right)\left(c\left(\sum_{l=1}^{s}(-1)^{\sigma_{l}} f_{j}^{(l)}\right)\right)^{2},
\end{aligned}
$$

так что

$$
\begin{aligned}
& \ln \frac{1-e^{-\left(R_{j}+1\right) b j}}{1-e^{-b j}}+\frac{c^{2}\left(\sum_{l=1}^{s}(-1)^{\sigma_{l}} f_{j}^{(l)}\right)^{2}}{2} \min _{x \in[b j / 2,3 b j / 2]} F^{\prime \prime}(x) \\
& \leqslant \phi_{j k} \leqslant \ln \frac{1-e^{-\left(R_{j}+1\right) b j}}{1-e^{-b j}}+\frac{c^{2}\left(\sum_{l=1}^{s}(-1)^{\sigma_{l}} f_{j}^{(l)}\right)^{2}}{2} \max _{x \in[b j / 2,3 b j / 2]} F^{\prime \prime}(x)
\end{aligned}
$$

(мы воспользовались неравенством (98)).

Прямое вычисление дает

$$
F^{\prime \prime}(x)=\frac{e^{x}}{\left(e^{x}-1\right)^{2}}-\frac{\left(R_{j}+1\right)^{2} e^{\left(R_{j}+1\right) x}}{\left(e^{\left(R_{j}+1\right) x}-1\right)^{2}},
$$

так что

$$
\max _{x \in[b j / 2,3 b j / 2]} F^{\prime \prime}(x) \leqslant \max _{x \in[b j / 2,3 b j / 2]} \frac{e^{x}}{\left(e^{x}-1\right)^{2}}=\frac{e^{b j / 2}}{\left(e^{b j / 2}-1\right)^{2}}
$$

(ибо вычитаемое положительно, а функция

$$
\frac{e^{x}}{\left(e^{x}-1\right)^{2}}=\frac{1}{e^{x}-1}\left[1+\frac{1}{e^{x}-1}\right]
$$

монотонно убывает) и

$$
\min _{x \in[b j / 2,3 b j / 2]} F^{\prime \prime}(x) \geqslant 0
$$


в силу утверждения 19. Итак,

$$
\begin{aligned}
& \ln \frac{1-e^{-\left(R_{j}+1\right) b j}}{1-e^{-b j}} \\
& \quad \leqslant \phi_{j k} \leqslant \ln \frac{1-e^{-\left(R_{j}+1\right) b j}}{1-e^{-b j}}+\frac{\left(c\left(\sum_{l=1}^{s}(-1)^{\sigma_{l}} f_{j}^{(l)}\right)\right)^{2}}{2} \frac{e^{b j / 2}}{\left(e^{b j / 2}-1\right)^{2}} .
\end{aligned}
$$

Таким образом, мы видим, что $\left|\phi_{j k}\right|$ экспоненциально убывает при $j \rightarrow \infty$, а так как кратности $q_{j}$ растут степенным образом, ряды в (96) сходятся.

Теперь, пользуясь правым неравенством в (101), с учетом (96) и (100) получаем

$$
\begin{aligned}
\mathscr{N}(\Delta) \leqslant & \exp \left\{b M+\sum_{j=1}^{\infty} \ln \frac{1-e^{-\left(R_{j}+1\right) b j}}{1-e^{-b j}}+N^{\gamma} \ln 2\right. \\
& \left.-c \Delta+\frac{c^{2}}{2} \sum_{j=1}^{\infty} q_{j} \frac{\left(\sum_{l=1}^{s}(-1)^{\sigma_{l}} f_{j}^{(l)}\right)^{2} e^{b j / 2}}{\left(e^{b j / 2}-1\right)^{2}}\right\} \\
\leqslant & \exp \left\{b M+\sum_{j=1}^{\infty} \ln \frac{1-e^{-\left(R_{j}+1\right) b j}}{1-e^{-b j}}+N^{\gamma} \ln 2-c \Delta+c^{2} C \sum_{j=1}^{\infty} \frac{j^{d-1+2 \alpha} e^{b j / 2}}{\left(e^{b j / 2}-1\right)^{2}}\right\} \\
\leqslant & \exp \left\{b M+\sum_{j=1}^{\infty} \ln \frac{1-e^{-\left(R_{j}+1\right) b j}}{1-e^{-b j}}+N^{\gamma} \ln 2-c \Delta+c^{2} \widetilde{C} b^{-d-2 \alpha}\right\},
\end{aligned}
$$

где $C, \widetilde{C}$ - некоторые постоянные. (В этих выкладках мы воспользовались оценкой (88), формулой (70) для $q_{j}$ и леммой 23.)

Положим

$$
c=\varkappa b^{d / 2(1-\gamma)+\alpha} \sqrt{\ln N} \psi(N),
$$

где $\varkappa>0$ - достаточно малая положительная постоянная. Тогда неравенство (97) выполнено в силу условия (86). С учетом асимптотической оценки $N \asymp b^{-d}$ (см. (28)) и формулы (87) для $\Delta$ получаем

$$
N^{\gamma} \ln 2-c \Delta+c^{2} \widetilde{C} b^{-d-2 \alpha} \leqslant-N^{\gamma}\left[\ln 2-K_{1} \varkappa \ln N \psi^{2}(N)\left(1-\varkappa K_{2}\right)\right],
$$

где $K_{1}, K_{2}>0$ - некоторые постоянные. При достаточно малом $\varkappa$ получим

$$
\mathscr{N}(\Delta) \leqslant C_{k} \exp \left\{b M+\sum_{j=1}^{\infty} \ln \frac{1-e^{-\left(R_{j}+1\right) b j}}{1-e^{-b j}}\right\} N^{-k}, \quad k=1,2, \ldots
$$

(При $\gamma \neq 0$ вместо множителя $C_{k} N^{-k}$ можно в этой оценке поставить даже экспоненту $e^{-K_{3} N^{\gamma}}$.) Комбинируя (103) с оценкой $(57)$ для числа $\mathscr{N}(M)$, завершаем доказательство теоремы 22.

3.2. Доказательство теорем о концентрации. 1. Теорема (12) является частным случаем теоремы 22 при $\gamma=0$ (и соответственно $s=1$ ).

2. Покажем, как из теоремы 12 вытекают теоремы 10 и 11.

Для доказательства оценок (35) и (36) в теореме 11 достаточно положить соответственно

$$
f_{j}=\left\{\begin{array}{ll}
1, & j \leqslant l, \\
0, & j>l
\end{array} \quad \text { и } \quad f_{j}= \begin{cases}j, & j \leqslant l, \\
0, & j>l .\end{cases}\right.
$$


Теорема 11 тем самым доказана, а теорема 10 получается из теоремы 11 переходом к пределу при $l \rightarrow \infty$.

3. Докажем теорему 13. Прежде всего, отметим, что для неотрицательных функций (45) справедлива оценка

$$
\sup _{x \in \mathbb{R}_{+}} \sum_{l=1}^{s} f_{l}(x) \leqslant \sup _{|y| \leqslant \mu} \sum_{l=-\infty}^{\infty} e^{-(y-\mu l)^{2} / 2} \equiv C_{\mu}<\infty,
$$

где постоянная $C_{\mu}$ зависит только от $\mu$ (и, в частности, не зависит от $\left.M\right)$. Несложные технические выкладки теперь показывают, что при выполнении условий теоремы справедливо равенство

$$
\begin{aligned}
\sum_{l=1}^{s}\left|\left(f_{l}, \xi_{M}-\xi\right)_{L^{2}\left(\mathbb{R}_{+}\right)}\right| & \equiv \sum_{l=1}^{s}\left|\int_{0}^{\infty} f_{l}(x)\left(\xi_{M}(x)-\xi(x)\right) d x\right| \\
& =b^{d} \sum_{l=1}^{s}\left|\sum_{j=1}^{\infty} f_{j}^{(l)}\left(N_{j}-\bar{N}_{j}\right)\right|+O(b),
\end{aligned}
$$

где

$$
f_{j}^{(l)}=\frac{1}{b} \int_{b(j-1)}^{b j} f_{l}(x) d x
$$

а постоянная в $O(b)$ не зависит от $M$. Далее, в силу (104) числа $f_{j}^{(l)}$ удовлетворяют условиям теоремы 22 при $\alpha=0$. Применяя эту теорему к правой части (105) с учетом неравенства $\gamma<1-2 / d$, получаем, что для каждого фиксированного $M$

$$
\sum_{l=1}^{s}\left|\left(f_{l}, \xi_{M}-\xi\right)_{L^{2}\left(\mathbb{R}_{+}\right)}\right| \leqslant C b
$$

с вероятностью не меньшей, чем $1-C_{k} N^{-k}$ для всех $k$, где $C, C_{k}$ - постоянные, не зависящие от $M$. Далее, если $P_{M}$ - проектор на подпространство, порожденное набором функций (45), то (норма и скалярное произведения подразумеваются в $L^{2}\left(\mathbb{R}_{+}\right)$, а суммы по $l$ от 1 до $\left.s\right)$

$$
\left\|P_{M} \eta\right\|=\max \frac{\left(\sum_{l} \alpha_{l} f_{l}, \eta\right)}{\left\|\sum_{l} \alpha_{l} f_{l}\right\|}
$$

где супремум берется по всем ненулевым векторам $\alpha=\left(\alpha_{1}, \ldots, \alpha_{s}\right)$. При достаточно большом $\mu$ матрица Грама $G$ с элементами $G_{m n}=\left(f_{m}, f_{n}\right)$ будет иметь диагональное преобладание:

$$
2 \sqrt{2 \pi} \sigma=G_{m m} \geqslant \lambda \sum_{n \neq m}\left|G_{m n}\right|,
$$

где $\lambda<1$ зависит только от $\mu$. Тогда, в силу свойств матриц с диагональным преобладанием,

$$
\left\|\sum \alpha_{l} f_{l}\right\|^{2}=(\alpha, G \alpha) \geqslant(1-\lambda) \sum_{l} G_{l l} \alpha_{l}^{2} \geqslant 2 \sqrt{2 \pi}(1-\lambda) \sigma \max _{l} \alpha_{l}^{2} .
$$


С другой стороны,

$$
\left(\sum_{l} \alpha_{l} f_{l}, \eta\right) \leqslant \sum_{l}\left|\left(f_{l}, \eta\right)\right| \max _{l}\left|\alpha_{l}\right| .
$$

Подставляя (110) и (111) в (108), получаем, что если $\mu$ достаточно велико, то

$$
\left\|P_{M} \eta\right\| \leqslant K \frac{\sum_{l}\left|\left(f_{l}, \eta\right)\right|}{\sqrt{\sigma}}
$$

где постоянная $K$ зависит только от $\mu$. Поскольку $\sigma=N^{-\delta} \geqslant K_{1} b^{d \delta}$, мы комбинируем (112) с (107) и получаем

$$
\mathrm{P}\left(\left\|P_{M}\left(\xi_{M}-\xi\right)\right\|_{L^{2}\left(\mathbb{R}_{+}\right)} \leqslant K_{2} b^{1-d \delta / 2}\right) \geqslant 1-C_{k} N^{-k}
$$

при всех $k$. Так как $\delta<2 / d$, отсюда непосредственно следует утверждение теоремы 13.

\section{4. Вспомогательные результаты}

\section{1. Две технические леммы.}

Лемма 23. Пусть $l$-вещественное число, а $k, m$ - целые числа такие, что

$$
0 \leqslant k<m
$$

Рассмотрим суммы

$$
\Sigma_{ \pm}=\sum_{j=1}^{\infty} \frac{j^{l} e^{k b j}}{\left(e^{b j} \pm 1\right)^{m}}
$$

где $b>0$ - вещественный параметр. Тогда при $b \rightarrow 0$ справедливы соотношения

$$
\Sigma_{-} \asymp\left\{\begin{array} { l l } 
{ b ^ { - l - 1 } , } & { \text { если } l > m - 1 , } \\
{ b ^ { - l - 1 } | \operatorname { l n } b | , } & { \text { если } l = m - 1 , } \\
{ b ^ { - m } , } & { \text { если } l < m - 1 , }
\end{array} \quad \Sigma _ { + } \asymp \left\{\begin{array}{ll}
b^{-l-1}, & \text { если } l>-1, \\
|\ln b|, & \text { если } l=-1, \\
1, & \text { если } l<-1 .
\end{array}\right.\right.
$$

Напомним, что запись $A \asymp B$ означает, что частное $A / B$ ограничено сверху и снизу положительным постоянными.

ДокАЗАТЕЛЬСтво. Проведем доказательство для суммы $\Sigma_{-}$(которую будем обозначать далее просто через $\Sigma$ ). Рассуждение для суммы $\Sigma_{+}$вполне аналогично.

Умножая и деля $\Sigma$ на $b^{l+1}$, получаем

$$
\Sigma=\frac{1}{b^{l+1}} \sum_{j=1}^{\infty} b \frac{(b j)^{l} e^{k b j}}{\left(e^{b j}-1\right)^{m}} \equiv \frac{1}{b^{l+1}} \sum_{j=1}^{\infty} b F\left(x_{j}\right),
$$

где

$$
F(x)=\frac{x^{l} e^{k x}}{\left(e^{x}-1\right)^{m}}, \quad x_{j}=b j, \quad j=1,2, \ldots
$$

Иными словами (с точностью до множителя $b^{-l-1}$ ) сумма (115) есть интегральная сумма для интеграла от функции $F(x)$. Мы используем этот факт для оценки 
указанной суммы. (Здесь на первый взгляд напрашивается применение формулы Эйлера-Маклорена, но оно не приводит к успеху при отрицательных $l-m$, так что мы проведем оценки непосредственно.)

Существует не зависящее от $b$ число $x_{0}>1$ такое, что функция $F(x)$ монотонно убывает при всех $x \geqslant x_{0}-1$. Действительно, с учетом (114) функцию $F(x)$ можно представить в виде произведения

$$
F(x)=\frac{x^{l}}{e^{x}-1}\left(1+\frac{1}{e^{x}-1}\right)^{k}\left(\frac{1}{e^{x}-1}\right)^{m-k},
$$

в котором второй и третий сомножители монотонно убывают при всех $x$, а первый сомножитель - при $x \geqslant l$. (В последнем легко убедиться: производная первого сомножителя имеет вид

$$
\left(\frac{x^{l}}{e^{x}-1}\right)^{\prime}=\frac{e^{x}\left(l x^{l-1}-x^{l}\right)-l x^{l-1}}{\left(e^{x}-1\right)^{2}}
$$

и заведомо отрицательна при $x \geqslant l$.) Таким образом, можно взять

$$
x_{0}=\max \{2, l+1\} .
$$

Разобьем теперь $\Sigma$ на две суммы, полагая

$$
\Sigma=\frac{1}{b^{l+1}}\left[\sum_{x_{j} \in\left(0, x_{0}\right]} b F\left(x_{j}\right)+\sum_{x_{j} \in\left(x_{0}, \infty\right)} b F\left(x_{j}\right)\right] \equiv \frac{1}{b^{l+1}}\left[\Sigma_{1}+\Sigma_{2}\right] .
$$

Все слагаемые (а следовательно, и обе суммы) положительны. Поэтому можно каждую из них оценивать по отдельности.

Оценка суммы $\Sigma_{2}$. Начнем с оценки второй из сумм. На интервале $\left(x_{0}, \infty\right)$ функция $F(x)$ монотонно убывает, и поэтому

$$
\int_{x_{0}-1}^{\infty} F(x) d x \geqslant \Sigma_{2} \geqslant \int_{x_{0}+1}^{\infty} F(x) d x
$$

так что вне зависимости от соотношения между $l$ и $m$ всегда выполнена оценка

$$
\Sigma_{2} \asymp 1 \text {. }
$$

Оценка суммы $\Sigma_{1}$. На интервале $\left(0, x_{0}\right]$ имеем $F(x) \asymp x^{l-m}$ и, следовательно,

$$
\Sigma_{1} \asymp \widetilde{\Sigma}_{1} \equiv \sum_{x_{j} \in\left(0, x_{0}\right]} b x_{j}^{l-m} .
$$

Рассмотрим два случая.

Случай $l-m>0$. В этом случае функция $x^{l-m}$ возрастает и справедливы неравенства

так что

$$
\int_{0}^{x_{0}+1} x^{l-m} d x \geqslant \widetilde{\Sigma}_{1} \geqslant \int_{0}^{x_{0}-1} x^{l-m} d x
$$

$$
\Sigma_{1} \asymp 1
$$


Случай $l-m<0$. В этом случае функция $x^{l-m}$ убывает и справедливы неравенства

$$
2 \int_{b / 2}^{x_{0}} x^{l-m} d x \geqslant \widetilde{\Sigma}_{1} \geqslant \int_{b}^{x_{0}} x^{l-m} d x .
$$

Вычисляя интегралы, получаем

$$
\Sigma_{1} \asymp \begin{cases}1 & \text { при } l-m>-1, \\ |\ln b| & \text { при } l-m=-1, \\ b^{l-m+1} & \text { при } l-m<-1 .\end{cases}
$$

Комбинируя это с оценкой для $\Sigma_{1}$ и умножая на $b^{-l-1}$, получаем утверждение леммы.

ЛЕмма 24. Пусть $l$-вещественное число, а $k, m$ - целые числа такие, что

$$
0 \leqslant k<m \leqslant l .
$$

Далее, пусть заданы произвольные числа

$$
a_{j} \in[1, \infty], \quad j=1,2, \ldots
$$

Рассмотрим сумму

$$
\Phi=\sum_{j=1}^{\infty} \frac{\left(a_{j} j\right)^{l} e^{k b a_{j} j}}{\left(e^{b a_{j} j}-1\right)^{m}}
$$

где $b>0$ - вещественный параметр и подразумевается, что те члены в сумме, для которых $a_{j}=\infty$, опускаются. Тогда при $b \rightarrow 0$ справедлива оценка

$$
\Phi \leqslant C b^{-l-1}
$$

с некоторой постоянной $C$, не зависящей от выбора последователъности $\left\{a_{j}\right\}$.

ДокАЗАТЕЛЬСтво. Справедливо равенство

$$
\Phi=\frac{1}{b^{l+1}}\left[\sum_{x_{j} \in\left(0, x_{0}\right]} b F\left(a_{j} x_{j}\right)+\sum_{x_{j} \in\left(x_{0}, \infty\right)} b F\left(a_{j} x_{j}\right)\right] \equiv \frac{1}{b^{l+1}}\left[\Phi_{1}+\Phi_{2}\right],
$$

где $F(x)$ и $x_{j}$ задаются формулой $(116)$, а число $x_{0}$ то же, что и в доказательстве леммы 23. В силу условия (117) функция $\Phi(x)$ равномерно ограничена сверху при всех $x \in(0, \infty)$ :

$$
F(x) \leqslant F_{0}<\infty .
$$

Поэтому

$$
\Phi_{1} \leqslant \sum_{x_{j} \in\left(0, x_{0}\right]} b F_{0} \leqslant \frac{x_{0}}{b} b F_{0}=x_{0} F_{0} .
$$

Чтобы оценить сумму $\Phi_{2}$, заметим, что для входящих в нее слагаемых как точки $x_{j}$, так и точки $a_{j} x_{j} \geqslant x_{j}$ лежат на участке монотонного убывания функции $F(x)$, так что

$$
\Phi_{2}=\sum_{x_{j} \in\left(x_{0}, \infty\right)} b F\left(a_{j} x_{j}\right) \leqslant \sum_{x_{j} \in\left(x_{0}, \infty\right)} b F\left(x_{j}\right)=\Sigma_{2}
$$

где $\Sigma_{2}$ - величина, введенная в доказательстве леммы 23. Там же было доказано, что $\Sigma_{2} \asymp 1$. Таким образом, мы оценили $\Phi_{1}$ и $\Phi_{2}$ сверху постоянными, которые не зависят от выбора последовательности $\left\{a_{j}\right\}$. Подстановка этих оценок в формулу (118) для $\Phi$ завершает доказательство леммы. 


\section{2. Доказательство предложений 6 и 7 .}

ДоКАЗАТЕЛЬСтво ПРЕДЛОЖЕНИЯ 6. 1. Рассмотрим функцию $\Psi(b)$, задаваемую правой частью формулы (23):

$$
\Psi(b)=\sum_{j=1}^{\infty} j q_{j}\left[\frac{1}{e^{b j}-1}-\frac{R_{j}+1}{e^{\left(R_{j}+1\right) b j}-1}\right]=\sum_{j=1}^{\infty} \frac{j q_{j}}{e^{b j}-1}-\sum_{j=1}^{\infty} \frac{j q_{j}\left(R_{j}+1\right)}{e^{\left(R_{j}+1\right) b j}-1} .
$$

Применяя к каждому из двух рядов в правой части лемму 23 , получаем, что

$$
|\Psi(b)| \leqslant C b^{-d-1} .
$$

С другой стороны, функция $F(a)=a\left(e^{a x}-1\right)^{-1}$ монотонно убывает по $a$ при любом фиксированном $x>0$ (чтобы в этом убедиться, достаточно вычислить ее производную). Поэтому

$$
\Psi(b) \geqslant \sum_{j=1}^{\infty} j q_{j}\left[\frac{1}{e^{b j}-1}-\frac{2}{e^{2 b j}-1}\right]=\sum_{j=1}^{\infty} \frac{j q_{j}}{e^{b j}+1} \asymp b^{-d-1}
$$

по лемме 23. В сочетании с предыдущим неравенством это показывает, что $\Psi(b) \asymp$ $b^{-d-1}$.

2. Покажем, что функция $\Psi(b)$ монотонно убывает при всех $b>0$. Достаточно продифференцировать отдельное слагаемое в сумме и убедиться, что производная отрицательна. Имеем

$$
\frac{\partial}{\partial b}\left[\frac{1}{e^{b j}-1}-\frac{R_{j}+1}{e^{\left(R_{j}+1\right) b j}-1}\right]=j\left[\frac{\left(R_{j}+1\right)^{2} e^{\left(R_{j}+1\right) b j}}{\left(e^{\left(R_{j}+1\right) b j}-1\right)^{2}}-\frac{e^{b j}}{\left(e^{b j}-1\right)^{2}}\right]<0
$$

(последнее неравенство получается, если применить утверждение 19 к выражению в квадратных скобках, которое представляет собой разность значений функции (77) в точках $R_{j}+1$ и 1 при $x=b j$ ).

Наконец, покажем, что функция $\Psi(b)$ принимает произвольно большие и произвольно малые положительные значения. Действительно, если для заданного $M_{0}$ взять $b>0$ настолько малым, что $e^{b M_{0}}<2$, то

$$
\sum_{j=1}^{\infty} \frac{j q_{j}}{e^{b j}+1} \geqslant \frac{M_{0}}{3}
$$

а значит, в силу (119) такое же неравенство верно и для $\Psi(b)$. При больших $b \gg 0$ запишем оценку

$$
\Psi(b) \leqslant \sum_{j=1}^{\infty} \frac{j q_{j}}{e^{b j}-1} \leqslant \sum_{j=1}^{\infty} \frac{j q_{j}}{e^{b j}}=e^{-b / 2} \sum_{j=1}^{\infty} \frac{j q_{j}}{e^{b(j-1 / 2)}} \leqslant e^{-b / 2} \sum_{j=1}^{\infty} \frac{j q_{j}}{e^{j-1 / 2}} .
$$

Последний ряд не зависит от $b$ и сходится. Поэтому $\Psi(b)$ произвольно мало́ при больших $b$. Предложение 6 доказано.

ДоКАЗАТЕЛЬСТво ПРЕДЛОЖЕния 7. Обозначим через $\widetilde{\Psi}(b)$ правую часть соотношения (27). Она отличается от $\Psi(b)$ только отсутствием множителя $j$ при квадратной скобке, что влияет на результат при применении леммы 23. В остальном доказательство вполне аналогично п. 1 доказательства предложения 6 . 
4.3. Доказательство формул с поправками для $M$ и $N$. В этом пункте мы дадим доказательство предложения 15.

Нужно вычислить суммы

$$
\sum_{j=1}^{\infty} \frac{j q_{j}}{e^{b j}-1}, \quad \sum_{j=1}^{\infty} \frac{q_{j}}{e^{b j}-1} .
$$

Вычислим прежде всего асимптотическое разложение для $q_{j}$ по степеням $j^{-1}$ :

$$
q_{j}=j^{d-1}\left(c_{0}+c_{1} j^{-1}+\cdots+c_{m} j^{-m}\right)+O\left(j^{d-m-2}\right) .
$$

Для вычисления коэффициентов $c_{m}$ воспользуемся рядом Стирлинга для $Г(z)$ при $z \rightarrow \infty:$

$$
\Gamma(z)=e^{-z} z^{z-1 / 2} \sqrt{2 \pi}\left(\sum_{k=0}^{n} a_{k} z_{-k}+O\left(z^{-n-1}\right)\right)
$$

где

$$
a_{k}=(2 k+1) ! ! b_{k},
$$

а $b_{k}$ определяются рекурсивно:

$$
b_{0}=b_{1}=1, \quad b_{k}=\frac{1}{k+1}\left(b_{k-1}-\sum_{j=2}^{k-1} j b_{j} b_{n+1-j}\right) .
$$

В частности,

$$
a_{0}=1, \quad a_{1}=\frac{1}{12}, \quad a_{2}=\frac{1}{288}, \quad a_{3}=-\frac{139}{51840}, \quad a_{5}=-\frac{571}{2488320} .
$$

Применяя формулу ряда Стирлинга к $\Gamma(j+d)$ и $\Gamma(j+1)$ в выражении $(20)$ для $q_{j}$, получаем асимптотическое разложение

$$
\begin{aligned}
q_{j} & =\Gamma(d)^{-1} \frac{e^{-j-d}(j+d)^{j+d-1 / 2}}{e^{-j-1}(j+1)^{j+1 / 2}} \frac{1+1 /(12(j+d))+\cdots}{1+1 /(12(j+1))+\cdots} \\
& =\frac{1}{\Gamma(d)}\left(\frac{j}{e}\right)^{d-1}\left(1+\frac{d}{j}\right)^{j+d-1 / 2}\left(1+\frac{1}{j}\right)^{-j-1 / 2} \frac{1+1 /(12(j+d))+\cdots}{1+1 /(12(j+1))+\cdots}
\end{aligned}
$$

(ошибка, возникающая при замене отношения гамма-функций его целой частью, поглощается остаточным членом). Отсюда получается искомое разложение по степеням $j^{-1}$. Вычислим для примера первые два члена. Справедливо равенство

$$
\left(1+\frac{x}{j}\right)^{j}=e^{x}\left(1-\frac{x^{2}}{2 j}+O\left(\frac{1}{j^{2}}\right)\right) .
$$

Поэтому

$$
\begin{aligned}
\left(1+\frac{d}{j}\right)^{j+d-1 / 2}\left(1+\frac{1}{j}\right)^{-j-1 / 2} & \\
& =\left(1+\frac{d}{j}\right)^{j}\left(1+\frac{1}{j}\right)^{-j}\left(1+\frac{d}{j}\right)^{d-1 / 2}\left(1+\frac{1}{j}\right)^{-1 / 2} \\
& =e^{d-1}\left(1-\frac{d^{2}-1}{2 j}+O\left(\frac{1}{j^{2}}\right)\right)\left(1+\frac{d(d-1 / 2)-1 / 2}{j}+O\left(\frac{1}{j^{2}}\right)\right) \\
& =e^{d-1}\left(1+\frac{d(d-1)}{2 j}+O\left(\frac{1}{j^{2}}\right)\right)
\end{aligned}
$$


Далее,

$$
\frac{1+1 /(12(j+d))+\cdots}{1+1 /(12(j+1))+\cdots}=1+O\left(\frac{1}{j^{2}}\right) .
$$

Окончательно получаем

$$
q_{j}=\frac{j^{d-1}}{\Gamma(d)}\left(1+\frac{d(d-1)}{2 j}\right)+O\left(j^{d-3}\right) .
$$

Теперь нужно подставить разложения (121) в (120) и затем вычислить суммы вида

$$
\sum_{j=1}^{\infty} \frac{j^{s}}{e^{b j}-1}
$$

Для этого воспользуемся формулой Эйлера-Маклорена

$$
\begin{aligned}
\sum_{j=1}^{n-1} f(j)= & \int_{0}^{n} f(x) d x-\frac{1}{2}[f(0)+f(n)]+\sum_{m=1}^{l-1} \frac{B_{2 m}}{(2 m) !}\left[f^{(2 m-1)}(n)-f^{(2 m-1)}(0)\right] \\
& -\frac{1}{(2 l) !} \int_{0}^{n} B_{2 l}(x-[x]) f^{(2 l)}(x) d x
\end{aligned}
$$

где $B_{k}$ - числа Бернулли, а $B_{k}(x)$ - многочлены Бернулли. В качестве функции $f(x)$ возьмем

$$
f(x)=\frac{x^{s}}{e^{b x}-1} \equiv b^{-s} F(b x), \quad F(x)=\frac{x^{s}}{e^{x}-1} .
$$

Возьмем максимальное $l$ такое, что $2 l<s+1$. Тогда интеграл в правой части равенства (124) абсолютно сходится и в нуле, и при $n \rightarrow \infty$, производные порядка $<2 l$ равны нулю в нуле, и поэтому, переходя к пределу при $n \rightarrow \infty$, получаем

$$
\sum_{j=1}^{\infty}[n-1] f(j)=\int_{0}^{\infty} f(x) d x-\frac{1}{(2 l) !} \int_{0}^{\infty} B_{2 l}(x-[x]) f^{(2 l)}(x) d x .
$$

Оценим последний интеграл. Имеем

$$
f^{(2 l)}(x)=b^{2 l-s} F^{(2 l)}(b x),
$$

так что

$$
\begin{aligned}
\left|\int_{0}^{\infty} B_{2 l}(x-[x]) f^{(2 l)}(x) d x\right| & \leqslant C b^{2 l-s} \int_{0}^{\infty}\left|F^{(2 l)}(b x)\right| d x \\
& =C b^{2 l-s-1} \int_{0}^{\infty}\left|F^{(2 l)}(x)\right| d x=C_{1} b^{2 l-s-1},
\end{aligned}
$$

где $C$ - максимум модуля многочлена Бернулли на отрезке $[0,1]$. С другой стороны,

$$
\int_{0}^{\infty} f(x) d x \equiv \int_{0}^{\infty} \frac{x^{s}}{e^{b x}-1} d x=b^{-s-1} \Gamma(s+1) \zeta(s+1),
$$

где $\zeta(s)$ - дзета-функция Эйлера. 
Итак, окончательно

$$
\sum_{j=1}^{\infty} \frac{j^{s}}{e^{b j}-1}=b^{-s-1} \Gamma(s+1) \zeta(s+1)\left(1+O\left(b^{2 l}\right)\right),
$$

где $l$ - максимальное целое число, такое, что $2 l<s+1$.

Подставляя теперь (123) в (120) и пользуясь (126), получаем формулы (51) и (52). Предложение доказано.

\section{СПИСОК ЦИТИРОВАННОЙ ЛИТЕРАТУРЫ}

[1] V.P. Maslov, On a distribution in frequency probability theory corresponding to the Bose-Einstein distribution, arXiv: math/0612394v1.

[2] V.P. Maslov, "Revision of probability theory from the point of view of quantum statistics", Russ. J. Math. Phys., 14:1 (2007), 66-95.

[3] В. П. Маслов, Квантовая экономика, Наука, М., 2007.

[4] В.П. Маслов, "Парастатистики и общая теорема теории вероятностей в приложении к безрисковым вложениям", Матем. заметки, 81:3 (2007), 478-480.

[5] V.P. Maslov, Quasithermodynamics and a correction to the Stefan-Boltzmann law, arXiv: $0801.0037 \mathrm{v} 1$.

[6] Л. Д. Ландау, И. М. Лифшиц, Статистическая физика, ГИТТЛ, М.-Л., 1951.

[7] В.П. Маслов, В.В. Вьюгин, "Теоремы о концентрации для энтропии и свободной энергии", Пробл. передачи информ., 41:2 (2005), 72-88.

[8] V.P. Maslov, Negative dimension in general and asymptotic topology, arXiv: math/ $0612543 \mathrm{v} 1$.

[9] М. В. Федорюк, Метод перевала, Наука, М., 1977.

[10] И.С. Градштейн, И. М. Рыжик, Таблищы интегралов, сумм, рядов и произведений, ГИФМЛ, М., 1963.

\section{В. П. Маслов}

Поступило

Московский государственный университет

11.01 .2008

им. М. В. Ломоносова

E-mail: v.p.maslov@mail.ru

\section{В. Е. Назайкинский}

Институт проблем механики РАН

E-mail: nazaikinskii@yandex.ru 